\title{
Change biases identify the features that drive time perception
}

\author{
Wouter Kruijne and Hedderik van Rijn \\ University of Groningen
}

\section{Author Note}

Wouter Kruijne (iD https://orcid.org/0000-0001-6358-7468,

Hedderik van Rijn (D) https://orcid.org/0000-0002-0461-9850

This work was financed by VICI grant 453-16-005 from the Netherlands Organisation for

Scientific research (NWO) awarded to HvR. Correspondence should be addressed to:

Wouter Kruijne, Department of Experimental Psychology, Faculty of Behavioral and Social

Sciences, University of Groningen, Grote Kruisstraat 2/1, 9712 TS Groningen.

E-mail: w.kruijne@rug.nl

Word Count: 10,519 


\begin{abstract}
Time perception is malleable, and the perceived duration of stimuli can be strongly affected by the sensory response they evoke. Such 'temporal illusions' provide a window on how different sensory systems contribute to our sense of time. Evidence suggests that the sensory response to different features affects time perception to different extents, mediated by the level of arousal or surprise that they evoke. This, however, makes it difficult to disentangle effects of the sensory response itself from the derived arousal or surprise effects. Here, we demonstrate that time perception is differentially affected by different stimulus features when arousal and surprise are kept constant. In four temporal discrimination experiments, participants were presented with empty intervals $(1.25-2.25 \mathrm{~s})$ marked by two briefly presented visual marker stimuli, and judged whether the duration was longer or shorter than a 1.75 s reference. Markers either repeated or changed along one of six feature dimensions, in a manner fully predictable to participants. Repetitions and changes would modulate sensory response magnitudes due to neural repetition suppression. Results showed that intervals were perceived as longer when markers changed in location, size or numerosity. Conversely, changes in face identity, orientation or luminance did not affect time perception. These results point to neural and functional selectivity in the way different stimulus features affect time perception.
\end{abstract}

Public significance statement: The neural sensory response evoked by a stimulus can affect its perceived duration. When a stimulus repeats, sensory neurons will have an attenuated response, which makes the stimulus seem to last shorter than when a stimulus changes. This study uses this observation to disseminate the relation between time perception and sensory responses to different visual features, and demonstrates that the different features that make up a stimulus affect its perceived duration.

Keywords: Time perception, Magnitude coding, Repetition suppression, Functional selectivity, Suprasecond durations, Visual perception 


\section{Change biases identify the features that drive time perception}

Perception is the process by which the brain transforms sensory information into a rich and meaningful experience. This process can be selective, as is the case when attentional mechanisms filter features or objects of the environment at the expense of other distracting information (e.g., Chun et al., 2011). Conversely, perception can be generative, which often takes on the form of illusions. In the case of the Kanizsa triangle (Kanizsa, 1955), for example, the perceived triangle is itself not present in the presented picture. Another example is the McGurk effect (McGurk \& MacDonald, 1976), where conflicting visual (/ba-ba/) and auditory (/ga-ga/) speech cues are integrated to give rise to a new perceived syllable (/da-da/). These illusions illustrate how perception can generate new meaning from objective sensory information. In that sense, the perception of time is one of the clearest demonstrations of generative perception. While visual and auditory perception have an objective physical origin, in the light that hits the cornea and the vibrations of the cochlear membrane, there is no sensory organ that directly detects the passage of time. And yet, even without such an organ we are able to perceive the duration of a range of stimuli and events under widely varying circumstances. For example, there is an immense difference in sensory input when waiting for the light to turn green at a busy intersection compared to waiting for an orchestra to start playing after the audience has gone silent in anticipation. Nevertheless, both these experiences can be perceived to have the same duration, and we are certainly able to tell when either of them takes unreasonably long.

Like most forms of perception, time perception is imperfect, which is why in daily life we often resort to standardized wall clocks and stopwatches to aid us in our chronometry. Many of the faults in time perceptions are systematic and can be used to inform and constrain models of time perception. For example, the 'scalar property' of timing refers to the empirical observation that the standard deviation of errors in perceived time grows linearly as a function of the observed duration. This is often considered a 'hallmark' of interval timing (e.g. Buhusi \& Meck, 2005, Okamoto \& Fukai, 2001, Pérez \& 
Merchant, 2018) that many models of time perception aim to account for: In most models the scalar property derives from some cumulative error in a sequentially updating dynamic timing computation (Gibbon, 1977; Simen et al., 2011; Oprisan \& Buhusi, 2014; van Rijn et al., 2014 but see Shankar \& Howard, 2011). Another class of systematic timing errors arise when memory traces of past intervals affect temporal percepts (Acerbi et al., 2012; Lejeune \& Wearden, 2009, Maaß et al., 2019, Vierordt, 1868). This phenomenon, known as Vierordt's law has been modeled extensively in recent Bayesian (Jazayeri \& Shadlen, 2010; Petzschner \& Glasauer, 2011; Sohn et al., 2018) or memory-pool frameworks (Bausenhart et al., 2014, Taatgen \& van Rijn, 2011).

However, there is another class of errors in time perception that do not result from the current or past duration of presented stimuli, but rather by nontemporal properties of the stimulus itself. These 'temporal illusions' often take on the form of systematic biases, leading certain stimuli to be consistently perceived longer or shorter than others. Such biases point to how sensory and temporal processing are not independent stages of perception, but that sensory information can causally affect time perception. This can be due to the physical properties of stimulus, such as how bright or loud stimuli are perceived as lasting longer than dimmed or softer stimuli (Goldstone et al., 1978; Zelkind, 1973, see also Ekman et al., 1969; Xuan et al., 2007, for other modalities). Alternatively, temporal illusions can be elicited by the context that a stimulus appears in: a range of experiments have demonstrated 'oddball' effects on time perception, where in a sequence of 'standard' stimuli, the duration of rare, deviant stimuli is often overestimated (Pariyadath \& Eagleman, 2008; Sadeghi et al., 2011; Tse et al., 2004; Ulrich et al., 2006).

One particular framework that has been proposed to account for such illusions is the magnitude coding hypothesis, which has been applied to a wide range of temporal bias effects (Eagleman, 2008; Matthews et al., 2014). The framework states that the magnitude of the neural response evoked by a stimulus will positively affect the perception of its duration. This explains why certain physical stimulus properties affect time perception, as 
these properties, like brightness or loudness, are known to produce larger responses in sensory neurons. In a similar vein, magnitude coding can account for the perceived dilation of the duration of oddball stimuli, as predictive coding theories prescribe that the unexpected deviant oddball will yield a larger cortical response compared to the often-repeated predictable standard (Clark, 2013, Friston, 2005, Rao \& Ballard, 1999; Stefanics et al., 2014). Furthermore, neurophysiological findings by Mayo and Sommer (2013) align with the magnitude coding account: They recorded neurons in the macaque frontal eye fields while the animals timed the interval between two flashing visual markers. Under- or overestimations of durations were found to be reflected in the amplitude of the visual response to the offset marker, with higher amplitudes for overestimated durations, and lower amplitudes for underestimated durations. Of note, the latency of these visual responses did not seem to be related to the perceived duration. Thus, the monkeys' subjective experience of time was seemingly not driven by the objective timing of the visual response, but was instead modulated by the amplitude of this sensory signal.

Although the magnitude coding framework fits with a range of empirical observations, it also has shortcomings that might have prevented it from being widely adopted into models of timing thus far. One particular shortcoming is that many of these findings do not exclusively support the magnitude coding account, and can instead also be explained in another manner. The most prominent alternative explanation is that temporal illusions might not be caused by the representation of the stimulus itself, but rather by the level of arousal or surprise that it evokes. This account derives from the well-established notion that time perception is modulated by the activity in dopaminergic neurons (Coull et al., 2011; Matell et al., 2014; Meck, 1986), and that midbrain dopaminergic activity is modulated by physical stimulus salience, surprise and the violation of expectations, arousal, and attention (Cho et al., 2017; Fiorillo et al., 2013; Meck, 2006; Schultz, 2016; Schultz et al., 1997). The causal effect of dopaminergic activity on time perception has strong neurophysiological support (Fung et al., 2021; Lake \& Meck, 2013; Soares et al., 2016) and 
presumably results from a speed-up of cortical dynamics (Mikhael \& Gershman, 2019, Paton \& Buonomano, 2018). Because of this relationship between arousal, dopamine and time perception, a range of temporal biases have been ascribed to increased levels of arousal (e.g., Droit-Volet \& Meck, 2007; Penton-Voak et al., 1996; Wearden et al., 2017). This also applies to many temporal illusions that have been taken to support the magnitude coding hypothesis: For example, 'brighter' or 'louder' stimuli can be regarded as more salient and thereby more arousing, and deviant stimuli in an oddball paradigm are more arousing since they violate expectations (Ernst et al., 2017; Tse et al., 2004; Ulrich et al., 2006). In fact, the magnitude of neural responses often is found to bidirectionally covary with arousal and surprise (Buia \& Tiesinga, 2006; Mather et al., 2016), and the effects of stimulus repetitions and switches on time perception are modulated by how predictable they are (Matthews, 2015; Wehrman et al., 2018). Other results, conversely, suggest that the physical properties of a stimulus may have effects on its perceived durations that run counter to the reported subjective arousal that this stimulus evokes (Thönes et al., 2018). Due to this convolution, it has proven difficult to establish to what extent time perception is affected by the magnitude of sensory responses directly, without modulations via arousal or surprise.

Recently, we have presented an experimental paradigm that might overcome this convolution (Kruijne et al., 2021). In this study, participants timed intervals that were presented by means of briefly flashing on- and offset markers (cf. Matthews, 2011a; Mayo \& Sommer, 2013), presented either left or right of fixation. The location of the onset marker varied across trials, and was therefore equally unpredictable and surprising across trials. Crucially though, participants knew on each trial whether the offset marker was going to be a repetition or a change with respect to the onset marker. As such, the offset marker was always fully predictable, regardless of whether it repeated or switched. Under such fully predictable conditions, neural responses to repeating stimuli are attenuated due to repetition suppression in a manner that is dissociable from suppression based on expectations (Grill-Spector et al., 2006, Larsson \& Smith, 2012, Todorovic \& Lange, 2012). 
As such, the design allowed us to study the effects of neural response magnitude on time perception, with minimal differences in salience, surprise, or arousal. The results showed that intervals were perceived as longer on trials where the marker changed, a phenomenon we called the 'change bias'. This bias indicated that duration perception was indeed affected by neural response magnitude and repetition suppression, without modulations by arousal or surprise. This conclusion was further supported by physiological signatures in event-related potentials and pupillometry. Components related to the sensory neural response were larger on change-trials than on same-trials, and their magnitude was predictive of the upcoming temporal judgment. Other physiological signatures related to surprise and arousal were not modulated by the predictable change (in direct contrast with neural markers of surprise found in temporal oddball paradigms: Ernst et al., 2017). Therefore, we argued that the change bias paradigm could offer a way to study the effects of neural repetition suppression on timing, and thereby test the magnitude coding hypothesis, with little to no interference from surprise and arousal.

Despite the physiological support for a link between change bias effect and the magnitude of sensory responses, it may be premature to equate these findings to magnitude coding effects reported in previous studies. For example, most of these (Eagleman \& Pariyadath, 2009; Matthews, 2015; Noguchi \& Kakigi, 2006; Pariyadath \& Eagleman, 2008) have used filled intervals where the interval is presented using a single stimulus, rather than an empty interval with briefly presented markers (though see Matthews, 2011a Mayo \& Sommer, 2013). If the change bias effect truly is an expression of magnitude coding, then the effect should not be limited to changes in 'location' as was manipulated in our previous work. Rather, one would predict that change biases in time perception would also arise with changes in other stimulus dimensions. This prediction is the impetus for the present study, where we investigate whether changes in different stimulus dimensions affect perceived durations. However, this prediction exposes a second shortcoming of the magnitude coding hypothesis: while the account posits a causal relation 
between time perception and the magnitude of neural responses, it does not specify which neural pathways implement this relation. A strong reading of the magnitude coding framework would suggest that this relation holds as a general rule across sensory neurons. On the other hand, it is possible that cortical neurons will display a degree of functional selectivity. Such selectivity would imply that neural responses in some areas might not affect the perception of time, even when they are attenuated by repetition suppression. There is tentative empirical evidence for such selectivity: Schindel et al. (2011) reported that oddball effects on time perception are absent when the oddball stimulus was similar to standards except that it was presented to a different eye. This change in the eye of origin would certainly have affected neural responses in striate visual cortex, suggesting that these neurons did not strongly affect time perception. Similarly, Mayo and Sommer (2013) reported signatures of magnitude coding in neurons in the frontal eye fields, but noted that these signatures were absent in visual neurons recorded in the superior colliculus. Here, we hypothesize that such neural selectivity could translate to functional selectivity. That is, different sensory stimulus features will have differential neural magnitude effects in different sensory areas. As a result, different stimulus features might have different effects on time perception, as the affected sensory areas may or may not be involved in constructing a temporal percept. Consequentially, it might be possible to use the change bias effect as a tool to identify stimulus features that play a causal role in the computation of a temporal percept.

Identifying the feature dimensions that drive the perception of time can help bridge the gap between, on the one hand, a range of behavioral biases on time perception, and on the other hand extensive neuroimaging work that has sought to identify the neural pathways involved in time perception and timed behavior (e.g., Bueti et al., 2008, Wiener et al., 2010). These neuroimaging efforts point to a vast network of areas involved in timing tasks including sensory cortex, parietal cortex, (pre-)motor cortex, the striatum, the thalamus, cerebellum, and the hippocampus. In recent years, more advanced analysis 
techniques and the usage of $7 \mathrm{~T}$ fMRI have made it possible to further partial out such networks into topographic maps, where different adjacent neural populations selectively respond to different intervals. Such chronotopic maps were first identified in the supplementary motor area and in the intraparietal sulcus (Protopapa et al., 2019). Recently, Harvey et al. (2020) presented evidence indicative of a hierarchically organized network of maps that range from from dorsal to anterior cortical areas. Interestingly, the regions that were identified as part of this network overlap largely with similar topographical networks that encode size and numerosity (Harvey et al., 2013; Harvey et al., 2015). These findings relate to a longstanding hypothesis that there is a shared neural representation for quantities or magnitudes in different stimulus dimensions, including duration, size and numerosity, where the parietal cortex is believed to play a central integrative role (Bueti \& Walsh, 2009; Bueti et al., 2007; Dehaene \& Brannon, 2010, Kanayet et al., 2017; Lambrechts et al., 2013, Martin et al., 2017; Walsh, 2003).

Here, we present four experiments where we use the change bias paradigm to study how repetitions or changes in different visual stimulus dimensions affect human time perception. The experiments served to investigate three primary research questions: First, we sought to demonstrate whether the change bias effect generalizes to changes in different feature dimensions. The results suggest they indeed do, which strengthens our interpretation that the change bias is a manifestation of how repetition suppression affects perceived time. Second, we investigated whether we can also identify visual features that are known to evoke repetition suppression effects, yet do not show change bias effects. We found this to be the case, which is indicative of both functional and neural selectivity in the visual features that drive the perception of time. Third, we explored this selectivity further, and tested to what extent such functional selectivity aligns with the neuroimaging results outlined above regarding quantity coding. In particular, we found that change bias effects on duration perception were found with visual stimuli that differed in location, size and numerosity, but not with any of the other tested feature dimensions. 


\section{Experiment 1 : Location and Face identity}

In an earlier study we have demonstrated that the change bias manifests when stimuli marking the start and end of an interval either repeat or change in location (Kruijne et al., 2021). The results suggested that sensory responses to the offset marker were attenuated upon repetition compared to changes, which in turn affected the perceived duration. Stimulus location is, however, not a feature dimension that is typically varied in studies that investigate repetition suppression. Many seminal studies have focused on the effects of the repeating versus changing faces (Henson, 2016; Ishai et al., 2006; Larsson \& Smith, 2012; Summerfield et al., 2008; Summerfield et al., 2011). Matthews (2015) used repeating and changing faces to show differential effects of expected and unexpected repetitions on time perception. We therefore first investigated whether the change bias generalizes to changes and repetitions of the identity of faces.

In Experiment 1 we presented participants with a typical temporal discrimination task (similar to Kruijne et al., 2021, Experiment 2) with three different block types: a block where face identity could change or repeat; a block where stimulus location could change or repeat, which served as a control condition and a replication of our earlier findings; and a block where on change-trials both stimulus location and face identity changed, which served to explore potential interaction effects between both dimensions.

\section{Method}

Participants. The study protocol was approved by the Ethical Committee of the Faculty of Behavioral and Social Sciences of the University of Groningen (EC BSS; internal review code PSY-1819-S-0201). 37 participants were recruited from the faculty participant pool and were compensated for their participation by means of course credit. Participants were treated in line with the guidelines in the Helsinki declaration, and gave written informed consent before participating. Six participants were excluded after preliminary analyses due to poor overall performance (details below), resulting in a sample of 31 
participants (26 female, ages 18-22, Mean age $=19.1$ )

\section{Figure 1}

Schematic illustration of the design and analysis of all experiments

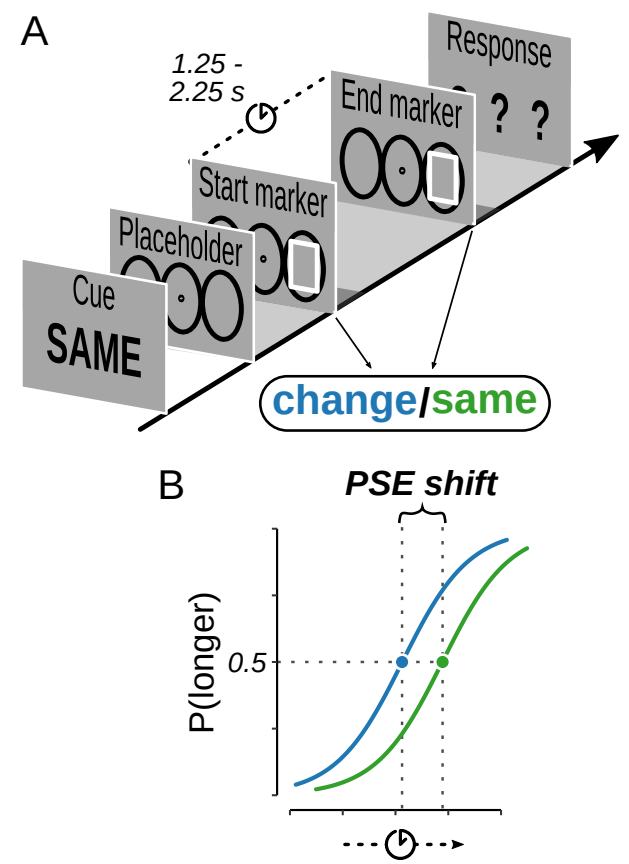

Note. A General trial sequence, here depicting a same-trial in a Location-block in Experiment 1. Participants were cued regarding the upcoming trial type (same or change). Their task was to estimate the interval between two flashing markers, which could either be the same or constitute a change. Subsequently, participants judged whether this was shorter or longer than a reference duration (1.75s). The precise timing of the other stimuli (for example, between Placeholders and Start marker, or between End marker and the Response) was fixed within but varied between experiments. See text for details. B The change bias is marked by a higher rate of 'longer' responses on change-trials. Response data were fit using logistic regression. The primary analysis captures these responses in different blocks in a single, hypothesis driven GLMM. In a complementary, more data-driven analysis the bias is quantified and analyzed as a shift in the Point of Subjective Equality (PSE).

Stimuli and Materials. Stimuli were presented on an LG-22MB37 LED monitor with $100 \mathrm{~Hz}$ refresh rate controlled by an HP Compaq Elite $3800 \mathrm{PC}$ with a $3.3 \mathrm{GHz}$ Intel 
i3-3220 processor. Participants were seated comfortably, at approximately $70 \mathrm{~cm}$ viewing distance from the screen. The experiment was programmed and run using OpenSesame (Mathôt et al., 2012) version 3.2.8 using the PsychoPy (Peirce, 2007) back-end. Stimuli were presented on a gray background with a black central fixation dot (radius $0.28^{\circ}$ ), and on each trial three black open placeholder circles (radius $4.44^{\circ}$; thickness $0.28^{\circ}$ ) were presented on the left, center, and right side of the screen to indicate where stimuli could appear. On each trial (Figure 1A), participants assessed the interval between two flashing marker stimuli and compared the interval between them to a reference interval $(1.75 \mathrm{~s})$. These stimuli, the start- and end-marker, could either be the same or could be different, and the feature dimension along which they varied was manipulated across blocks. In Face-blocks, each marker would be a picture of a face (detailed below) presented in the center placeholder. In Location-blocks, markers were presented as a white open square, presented either on the left or right. In Both-blocks, markers were faces that were either on the left or on the right. The reference interval was presented in similar fashion, by means of white open squares in the center of the screen.

The white square stimulus, used in Location-blocks and reference trials, had $6.11^{\circ}$ sides and fit exactly within a placeholder circle. Face stimuli, used in Face- and Both-blocks, were drawn from 733 pictures of faces that were extracted from several face datasets: 115 from the Psychological Image Collection at Stirling (pics.stir.ac.uk; Pain, Utrecht- and Stirling- datasets); 40 from the ATET labs database of faces (Samaria \& Harter, 1994); 26 from the Caltech faces database (www.vision.caltech.edu/html-files/archive.html); 28 from the BioID face database (www.bioid.com/facedb/); 38 from the Extended Yale Face Database B (Georghiades et al., 2001); 485 from the Labeled faces in the wild (LFW) database (Learned-Miller et al., 2016). Images from these databases were filtered, manually discarding duplicate identities, images with poor quality, familiar subjects, (partial) face occlusions, faces explicitly labeled as not 'neutral', and other artifacts. A complete list of the final 733 set of images is available at 
https://osf.io/e89hk/. The faces within these images were localized and extracted using a face detection algorithm implemented in OpenCV, such that for each base image a face image was extracted of $175 \times 175 \mathrm{px}$ in grayscale (256-bit png format). From the final set of 733 face images, faces were randomly sampled for each participant such that no two trials would use the same face. Images were scaled to have the same size as the white square $\left(6.11^{\circ}\right)$, and a white squared frame was added around it to render the overall stimulus brightness comparable across conditions.

Design and procedure. Here, we first outline the overall structure of the experiment, followed by a description of the procedure on individual trials. At the start of the experiment, participants were informed that they were going to be presented with different intervals, which they had to compare to a reference interval. This reference interval $(1.75 \mathrm{~s})$ was then presented nine times, using a white, central square as both onsetand offset markers. Participants were asked to memorize this duration. Subsequently, they completed a practice block of 10 trials presented in a similar fashion, with intervals of a randomly sampled duration between 1.25 and $2.25 \mathrm{~s}$ which they had to judge as 'shorter' or 'longer' than the reference. During practice, participants received feedback on every trial. After practice, they were informed that there would be three different block types in the experiment (Face/Location/Both) which differed only in the marker stimuli used to present intervals (Figure 2A). They were also informed that stimuli could repeat or change within a trial, as indicated by a cue. They then completed twelve blocks of 40 trials each. The three block types were presented as triplets, with four triplets making up the entire experiment. The order of blocks within each triplet was random. At the start of each block, participants were shown the reference interval three times as a reminder, and were informed of the upcoming block type. Between blocks, participants were given the opportunity to rest, and continue at their own pace. The entire experiment took approximately 50-60 minutes.

On each trial, participants were first shown a cue indicating the trial type (same/change), by means of the word SAME or CHANGE presented in the center of the 
screen for $400 \mathrm{~ms}$. This cue was valid, which made both repetitions and changes in stimulus marker fully predictable. However, regardless of the cue they could not predict the nature of the onset marker: only that this stimulus would repeat or change in the later offset marker. The fixation dot appeared immediately after the cue, and was after $500 \mathrm{~ms}$ followed by the onset of the placeholder circles which stayed on screen until the end of the trial. After another 500ms, the start marker appeared on screen for $200 \mathrm{~ms}$ and then disappeared, followed by the presentation of the offset marker for another $200 \mathrm{~ms}$. The stimulus onset asynchrony between both markers was randomly sampled between 1.25 and $2.25 \mathrm{~s}$, and served as the interval presented on that trial. The reference- and practice trials were presented in the same way, but using a blank screen in place of the word cues. Participants were instructed to compare the time between the onsets of both markers to the reference interval $(1.75 \mathrm{~s})$, and to give a non-speeded response indicating whether the presented interval had been shorter $(\mathrm{F})$ or longer $(\mathrm{J})$. To discourage speeded responses and to stress accuracy, the end marker was followed by a response delay of 500ms during which only the placeholders were visible. After this delay the placeholders disappeared and participants could respond. When a response was made, the trial ended and the next trial began.

Statistical Analyses. All analyses were conducted in R (R Core Team, 2020). Participants' performance was initially assessed by means of a logistic regression on all trials, irrespective of condition: $P($ longer $)=\operatorname{logit}\left(\beta_{0}+\beta_{\Delta t} \cdot \Delta t\right)$, where $\Delta t=t-1.75 \mathrm{~s}$, the deviation of the presented interval in seconds with respect to the reference duration. The slope coefficient $\beta_{\Delta t}$ was transformed to a just noticeable difference score (JND, also sometimes known as the difference limen) via $J N D=\ln (3) / \beta_{\Delta t}$. This JND estimates the duration difference between $25 \%$ and $75 \%$ 'longer' responses. One interpretation of this measure is that it marks the interval range over which participants were largely unsure about their responses. Following this interpretation we excluded participants where $J N D>0.5 s$ (six participants, mentioned above as having 'poor performance').

Our primary analysis focused on evaluating the change bias (Figure $1 \mathrm{~B}$ ) in the three 
block types. We took two complementary approaches to statistically assess this question. Leading in our analyses was a hypothesis-driven mixed-modeling approach, which utilizes data across all blocks and exploits shared hierarchical variance across participants to produce accurate psychometric fits to the data and to assess any shifts across conditions therein. Complimentary to this approach was a data-driven assessment, based on individual psychometric curves fit separately for each participant and each condition. This approach allows insight into the variability of change biases across participants and stimulus conditions.

For the hypothesis-driven tests, we fit a general linear mixed effects model (GLMM; Baayen et al., 2008) to the data, using a logistic link function. This model included the fixed effects terms 'interval duration' ( $\Delta t$ in seconds with respect to the reference interval), 'block type' (Face/Location/Both), 'trial type' (same/change), and an interaction term 'block type $\times$ trial type' to allow effects of stimulus changes to vary across block types. Additionally, the model included uncorrelated random effects terms to estimate hierarchical variance in the intercept and the slope coefficients of the psychometric curves. In all experiments this was the preferred random effects structure based on the Akaike Information Criterion (AIC). Within this GLMM, we tested for significant differences between same- and change-trials in all three block types separately, using Tukey HSD tests on the estimated marginal means of the model (using package 'emmeans'; Lenth, 2020).

This hypothesis-driven model makes several assumptions that were based on our earlier findings (Kruijne et al., 2021). Most importantly, the model assumes that experimental conditions (trial type and block type) only differ with respect to the shift of the psychometric curves, but not their slope. To test whether this assumption was valid, we conducted additional model comparisons between the hypothesis-driven model and more complex models that included terms that implemented interactions with 'interval duration' (in other words, allowing the slope of the psychometric curve to vary across block types and trial types). For these comparisons we report the difference in the Bayesian 
Information Criterion $(\triangle B I C)$ and report Bayes Factors (BFs) derived from these BICs (Wagenmakers, 2007). For completeness, we also report $\chi^{2}$ statistics and corresponding p-values from Likelihood Ratio Tests, although the more conservative Bayesian statistics will be leading for inferences concerning these exploratory analyses. Another assumption of the hypothesis-driven model is that responses approximately follow a logistic curve. Across all four experiments, however, we observed a tendency for the logistic fit to overestimate the proportion of 'longer' responses to longer interval durations, which is presumably due to the scalar property of timing. To ensure that critical statistical inferences were not affected by this subtle misfit, we also tested the same contrast in a GLMM where 'interval duration' was a categorical variable, expressed in four equally spaced duration bins (the same bins are used in Figures 2-5A to visualize average responses). This model has much more degrees of freedom and was therefore never preferred over the model with a continuous 'duration' effect, but it allowed us to specifically test the critical contrast between change- and same-trials across block types in a manner that is agnostic with respect to the precise shape of the psychometric curve.

For the data-driven assessment of the change-biases across participants and across conditions, we fit independent logistic psychometric curves separately for each participant, block type and trial type. From the coefficients of these fits we derived the point of subjective equality in each experimental condition $\left(P S E=-\beta_{0} / \beta_{\Delta t}\right)$, and quantified the change bias as $P S E_{\text {change }}-P S E_{\text {same }}$ for each block type. These biases were then subjected to Bayesian- and frequentist one-sided t-tests to assess whether this difference was greater than zero (using the 'BayesFactor' package; Rouder et al., 2009). This data-driven analysis has the additional benefit that it expresses the magnitude of the change bias on an intuitive scale (PSE shift in seconds), and that it gives insight into the variability of the bias effects across individual participants (Figures 2-5B).

For this experiment, as well as for Experiments 2-3, our analyses primarily focus on the contrast between the two trial types: same versus change. In doing so, we generally 
collapse across all stimulus pairs that are used to present either same- or change-trials. Additionally, we largely ignore the absolute PSEs that arise in these different stimulus conditions. The reasoning behind this is that any temporal biases that may emerge for different start- and end markers would affect same- and change-trials to the same extent. In a supplementary analysis (https://osf.io/e89hk/), we present additional, exploratory plots and analyses of the PSE of all stimulus pairs in each experiment, and present pairwise comparisons between the different stimulus pairs within each block type. As a general rule, we found that in all block types that gave rise to change biases, a similar bias was found across all stimulus pairs that constituted a repetition versus all pairs that implement a change. Furthermore, these analyses revealed that PSEs were highly variable across participants, indicating large trait-level biases in temporal judgments. Nevertheless, the analyses presented in the main text indicate that the change biases that affected these judgments were much more consistent across participants.

Sample size and power. Sample sizes were based on a GLMM fit to earlier results (Kruijne et al., 2021, Experiment 2) and were validated using power analyses with the 'simr' package (Brysbaert \& Stevens, 2018; Green \& MacLeod, 2016). We simulated 250 experiments with this model, based on the current number of trials per block type (160), using a conservative effect size estimate, four times smaller than what had been observed. Power was determined at different sample sizes by evaluating the probability of detecting a significant difference between trial types, at $\alpha=0.05$. Results indicated that the power estimate significantly exceeded $80 \%$ with a sample size of 25 participants or more (85.6\%, 95\% confidence interval: 80.6-89.7\% ). In all exeriments, we therefore collected data from cohorts of participants until the number of participants exceeded 25 after applying exclusion criteria. 


\section{Figure 2}

Stimuli and results in Experiment 1

\section{Experiment 1}

A
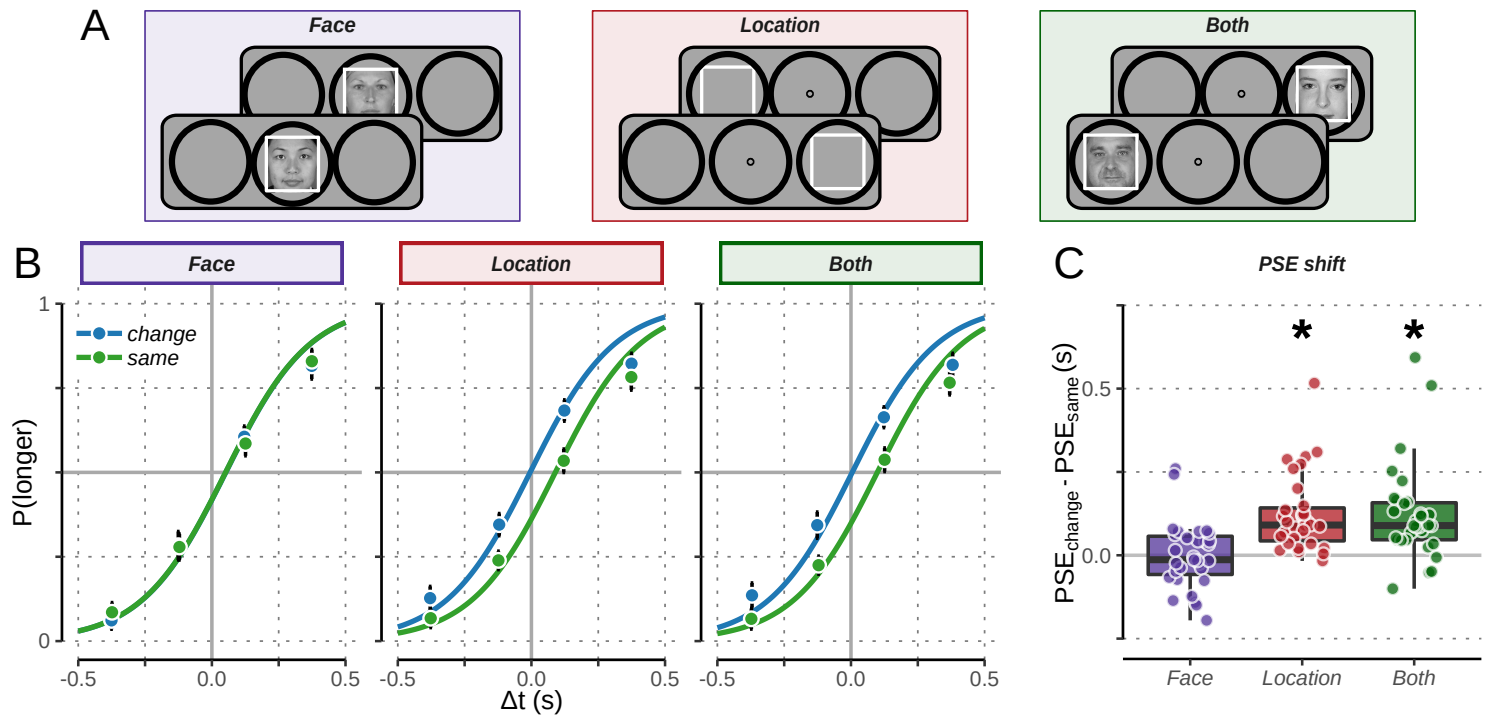

Note. A Schematic illustration of start- and end-marker stimuli in different block types. Faces were unique on each trial. B Psychometric curves for two trial types (same/change) in three block types (Face/Location/Both), derived from the hypothesis-driven GLMM. There is a clear change bias in Location- and Both-blocks, but not in Face-blocks. Data points depict average 'longer' response-rates across participants in four interval bins, with $95 \%$ Cousineau-Morey confidence intervals (Cousineau, 2005: Morey, 2008). C PSE-shifts from same- to change-trials for individual participants. Again, results revealed a positive shift in PSE in Location- and Both-blocks, but not in Face-blocks.

\section{Results}

The results of Experiment 1 are depicted in Figure 2B \& C. Data points in Figure $2 \mathrm{~B}$ depict the average proportion of 'longer'-responses in four interval bins, separately computed in for all trial type and block type conditions. Whiskers indicate $95 \%$ within-subject confidence intervals (Cousineau, 2005; Morey, 2008). The curves depict the fits of the hypothesis-driven GLMM, which reveal a change bias manifested as a 
psychometric curve shift, in Location- and Both-blocks but not in Face-blocks. This observation was supported by Tukey HSD tests which did not suggest a difference between same- and change-trials in Face blocks $\mathfrak{1}^{1}(z=-0.07, p=0.943)$, but did suggest a significant difference in Location-blocks $(z=8.06, p<0.001)$ and Both-blocks $(z=8.02, p<0.001)$. Analyzing the same contrast in the GLMM where 'interval duration' was coded by four factorial bins lead to the same conclusions (Face-blocks: $z=-0.26, p=0.794$; Location-blocks: $z=7.96, p<0.001$; Both-blocks: $z=7.87, p<0.001$ ).

Further model comparisons suggested that the hypothesis-driven model was preferred over more complex models. For example, we found strong evidence that the change bias did not differ across interval durations $\left(\triangle B I C=-5.96 ; 1 / B F=19.68 ; \chi^{2}(1)=3.65 ; p=0.056\right)$. We additionally found evidence that timing sensitivity, quantified by the slope of the psychometric curve, did not differ across block types $\left(\triangle B I C=-11.95 ; 1 / B F=393.47 ; \chi^{2}(2)=7.26 ; p=0.026\right)$. While not supported by Bayesian tests, the likelihood ratio test suggested potential slope differences across block types, with marginally better timing in Face-blocks, and subtly worse timing in Both-blocks (JND estimates from this more complex model:

$\left.J N D_{\text {Face }}=0.165 ; J N D_{\text {Location }}=0.175 ; J N D_{\text {Both }}=0.181\right)$.

Figure $2 \mathrm{C}$ shows the results from the data-driven analysis of psychometric curves, with individual data points depicting PSE shifts from change- to same-trials for each participant. The results are overall in line with the mixed-effects model analysis. One-sided t-tests of estimated PSE shifts gave strong evidence that there was no change bias in Face-blocks $(t(30)=0.06 ; p=0.524 ; 1 / B F=5.21)$, whereas a positive PSE shift was found in Location-blocks $(t(30)=5.88 ; p<0.001 ; B F>1000)$ and Both-blocks $(t(30)=4.70 ; p<0.001 ; B F=453.19)$. Pairwise comparisons of the PSE shifts across

\footnotetext{
${ }^{1}$ In these and all subsequent contrasts, we will use positive values for $z$ and $t$ to indicate that change-trials were judged 'longer' more often than same-trials, and negative values to indicate differences in the opposite direction.
} 
block types indicated that both Location- and Both-blocks showed a larger bias than Face-blocks (both $t(30)<-4.17 ; p<0.001 ; B F>118.83$ ) but the magnitude of the change bias did not differ across these two block types $(t(30)=-0.08 ; p=0.940 ; B F=5.21)$.

\section{Discussion}

The results of Experiment 1 replicate our earlier findings: A change in marker locations makes the interval denoted by them seem to last longer. However, we found no change bias in Face-blocks where markers constituted a change in face identity without changing location; neither in the hypothesis-driven GLMM nor in the data-driven PSE analysis. Extensive neuroimaging research has shown that repetitions and changes in face identities under very similar conditions give rise to repetition suppression effects in cortical areas tuned to faces. Therefore, the results of Experiment 1 suggest that repetition suppression due to face repetitions does not affect time perception in the way that repetitions and switches in locations do. The results therefore do not support the strong reading of the magnitude coding hypothesis that neural magnitude in sensory neurons universally affects time perception. However, it still leaves the possibility that these discrepant results are a consequence of selectivity in the sensory neurons that have magnitude coding effects on perceived time. In Experiments 2-4, we therefore tested changes in other stimulus dimensions to assess whether they affect time perception.

\section{Experiment 2 : Location and Size}

Experiment 2 sought to investigate whether the change bias effect can be evoked by changes in stimulus size. Size is a feature that is often considered in quantity coding theories (Bueti \& Walsh, 2009; Walsh, 2003), where it is proposed to have a shared neural representation with other quantities including duration. These theories motivated the hypothesis that changes in stimulus size affect perceived duration much like changes in location. Furthermore, a previous study already suggested that changes in size can affect perceived durations (Matthews, 2011a). However, intervals in that study were much 
shorter $(<600 \mathrm{~ms})$ than the intervals used here, and repetitions and changes were not predictable. Therefore, it is possible that alternative explanations involving apparent motion or a violation of expectations may account for those results. Experiment 2 used a design closely resembling Experiment 1, and tested whether predictable changes in stimulus size gives rise to change biases in time perception.

\section{Methods}

The design, procedure and analyses of Experiment 2 were in almost all regards identical to Experiment 1, but replacing Face stimuli with a rectangle that could vary in size. In the following, we will only discuss the aspects in which both experiments differed.

Participants. The protocol for Experiment 2 was approved by the EC BSS (internal review code PSY-1819-S-0273). We recruited 29 participants, and discarded data from four due to poor performance. Analyses are thus based on data from 25 participants (20 female, ages 18-23, Mean age $=19.28)$.

Stimuli and materials. Stimuli and displays used in different block types are schematically depicted in Figure 3A. Due to the variable size of marker stimuli, Experiment 2 did not use placeholder circles to indicate where stimuli might appear. Instead, this was achieved by means of three fixation dots at possible stimulus locations. All interval markers (including those in reference trials) were dark green open squares (as in Kruijne et al., 2021), which were somewhat less straining on the eyes than the white squares used in Experiment 1. In Size-blocks, this square had sides of either $3.85^{\circ}$ or $11.4^{\circ}$, which constitutes a change of $\pm 50 \%$ with respect to its size in reference trials and Location-blocks $\left(7.67^{\circ}\right)$. In Location-blocks, the square could again be presented on the left or on the right, and in Both-blocks changes in these dimensions were combined so that a change in location was always accompanied by a change in size.

Design and Procedure. The presentation sequence of a single trial was identical to Experiment 1, other than that the marker stimuli were each on screen for only $125 \mathrm{~ms}$. 
The response delay was shortened to $400 \mathrm{~ms}$. The entire experiment took approximately 45-55 minutes.

Statistical analyses. The statistical procedure was identical to that of Experiment 1.

\section{Figure 3}

Stimuli and results in Experiment 2

\section{Experiment 2}
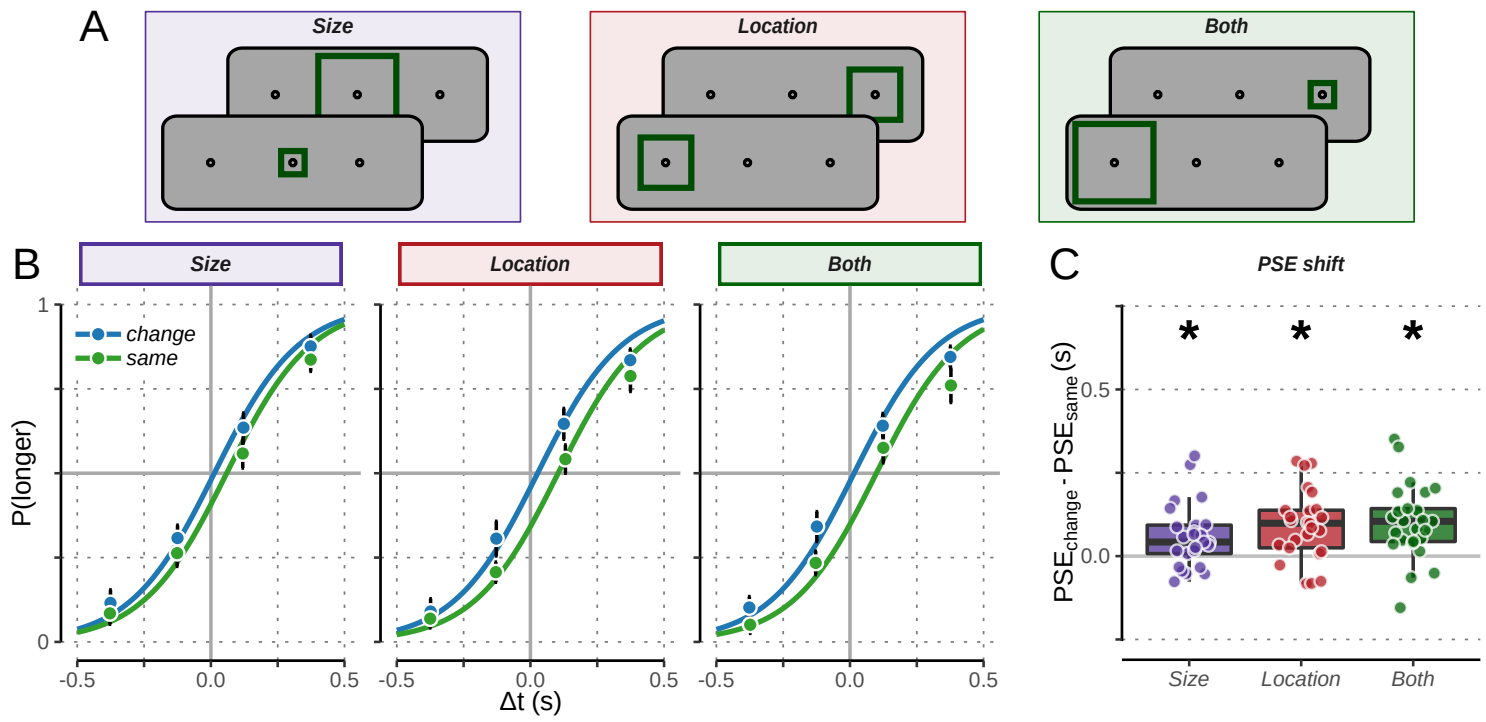

Note. cf. Figure 2, A Schematic illustration of the stimuli used as start- and end marker in different block types. B The GLMM fits indicate that a change bias manifested in Size-blocks, as well as in Location- and Both-blocks. C PSE-differences computed from individual psychometric curves show a positive shift in all three conditions.

\section{Results}

The results of Experiment 2 are depicted in Figure $3 \mathrm{~B} \& \mathrm{C}$. The fits of the hypothesis-driven GLMM (Figure 3B) illustrate a change bias effect in all three block types, marked by a PSE-shift between same- and change-trials. The Tukey HSD tests of the same-change contrast across block type indicated significantly more 'longer' responses 
to change-trials in all three block types (Size: $z=3.55, p<0.001$; Location: $z=5.88, p<0.001$; Both: $z=6.41, p<0.001)$. Again, analyzing this contrast in a GLMM where duration was coded as a factorial variable yielded the same conclusions (Size: $z=3.61, p<0.001$; Location: $z=5.60, p<0.001$; Both: $z=6.23 ; p<0.001)$. Model comparisons supported the hypothesis-driven model over more complex models. That is, there was strong evidence that trial type did not affect the slope of the psychometric curve $\left(\triangle B I C=-8.37 ; 1 / B F=65.68 ; \chi^{2}(1)=1.02 ; p=0.312\right)$, and that the slope did not differ across different block types $\left(\triangle B I C=-17.48 ; 1 / B F>1000 ; \chi^{2}(1)=1.23 ; p=0.523\right)$.

Figure $3 \mathrm{C}$ summarizes the data-driven analysis of the change bias as quantified by PSE-shifts estimated from individual psychometric curves. In line with the mixed-effects model analyses, the t-tests of these PSE-shifts suggested that in all block types the marker change gave rise to a positive shift in PSE, in Size-blocks $t(24)=3.09 ; p=0.003 ; B F=8.55)$, in Location-blocks $(t(24)=4.34 ; p<0.001 ; B F=133.46)$, and Both-blocks $(t(24)=4.51 ; p<0.001 ; B F=192.58)$.

Numerically, both the hypothesis driven GLMM-analysis and the data-driven PSE analysis suggested that a reliable change bias was found in all block types, but that it might have been larger in Both-blocks than in Location- and Size-blocks. We therefore ran follow-up analyses comparing different GLMMs to assess whether this was indeed the case, and to explore whether the bias in Both-blocks should perhaps be defined as an additive effect of a change bias caused by the combination of changes in size and location. To this end, we split the variable 'trial type' (same/change) into two separate predictor variables, one marking 'location changes' and one marking 'size changes'. We then tested whether the change bias was best captured by a model that had independent bias effects for each block type (the original model), a single change-effect that held across all block types, or additive change effects for location- and size-changes. Model comparisons indicated that the best model was one with a single change-effect capturing data in all blocks, omitting the 
interaction term $\left(\triangle B I C=-14.17 ; 1 / B F>1000 ; \chi^{2}(2)=4.62 ; p=0.099\right)$. This model was also strongly preferred over the 'additive change-effects' model $\left(\triangle B I C=-7.83 ; 1 / B F=50.09 ; \chi^{2}(1)=1.56 ; p=0.211\right)$.

These analyses indicate that the change bias was best captured by a model that describes the bias in a single term, with similar magnitude across block types. Therefore, we ran further exploratory analyses to investigate whether the change biases, as quantified by PSE-shifts in different block types, were correlated across participants. We found moderate evidence that this was indeed the case: (Size and Location: $r=0.41, p=0.041, B F=2.39$; Size and Both: $r=0.57, p=0.003, B F=15.41$; Location and Both: $r=0.44, p=0.030, B F=3.00)$.

\section{Discussion}

In Experiment 2, we again replicated the change bias effect caused by changes in location, but also found a similar bias evoked by changes in stimulus size. Similar to the findings of Matthews (2011a), we showed that a change in the size between two briefly presented squares makes the interval between them seem to last longer. We extended those findings by showing that this effect is consistent for changes across a range of supra-second intervals, and is found even when the change is fully predictable. These observations further support the hypothesis that temporal illusion caused by changes in marker size is due to magnitude effects in the sensory representation of the marker, rather than a result of apparent motion or a violation of expectations.

Our reason to assess the effects of size changes was in part motivated by theories that postulate a shared representation for visual 'magnitude' and 'space', which affect time perception and have been related to the parietal cortex. The results so far are in agreement with these theories: repetitions and changes of Size and Location were found to affect time perception, whereas changes in the identity of face stimuli do not. However, an alternative and perhaps simpler explanation would be that in our experiments, the feature changes 
that give rise to change bias effects are also associated with clear changes in low-level visual processing: due to the retinal displacement of the stimulus boundaries in both Sizeand Location blocks, the neural representation of a changing stimulus would have been very different. In the blocks in Experiment 1 where only the identity of the face changed, it might have been that the only substantial change in neural representation may have been in relatively late stages of visual processing, where the identity of the face is represented. As such, it is possible that the duration of the interval was already extracted before neural repetition suppression in face-selective areas had any effect.

To test the plausibility of this alternative explanation, Experiment 3 tested whether time perception would be affected by changes in the orientation of a Gabor-stimulus. The orientation of such stimuli is canonically associated with early low-level visual processing. Despite these low-level changes, changes in orientation are not reflective of a change in location or quantity, and have not been related to the postulated common quantity representation in parietal cortex.

\section{Experiment 3: Location and Orientation}

Experiments 1 and 2 showed that changes in different feature dimensions can have differential effects on temporal processing: Changes in location and size cause a lengthening of perceived duration, whereas changes in face identity showed no such effects. This discrepancy could be reflective of a shared common representation between space, time, and magnitude. On the other hand, it could also suggest that time perception is driven by activity in early vision, and that repetition suppression effects caused by face identity changes take place at a later stage, and therefore did not affect time perception. In Experiment 3 we aim to disentangle these two explanations by testing whether a visual feature that is not hypothesized to have a shared representation with space and magnitude, but is associated to early stages of visual processing does or does not affect temporal processing. To this end, we assessed the effects of changes in the orientation of rotated 
gabors. Gabor stimuli are typically associated with early-stage visual processing, and they have often been used to illustrate neural orientation tuning in striate visual cortex (Beck \& Kastner, 2007; Fang et al., 2005; Movshon \& Van Sluyters, 1981; Tootell et al., 1988). The neural effect of changes and repetitions in the orientation of such oriented gratings have been explored using MEG and fMRI (Hansen \& Dragoi, 2011; Kok et al., 2012; Tang et al., 2018), similarly pointing to pronounced effects in early visual processing. If such early effects of repetitions and changes on the magnitude of sensory responses would causally impact perceived durations, we would expect that changes in the orientation of gabor stimuli will also give rise to change biases, much like Location and Size.

\section{Methods}

The design, procedure and analyses of Experiment 3 were in almost all regards identical to Experiment 2, but replacing Size stimuli with gabor stimuli rotated $45^{\circ}$ clockwise or counterclockwise. Here, we will only describe the ways in which Experiment 3 differed from Experiment 2.

Participants. The protocol for Experiment 3 was approved by the EC BSS (internal review code PSY-1920-S-0190). We collected data from 40 participants but excluded three: two did not complete the experiment, and one took 20 minutes longer than the rest. Of the remaining participants, data from nine were discarded due to poor performance. The final data set included 28 participants (20 female, Ages 18-24, Mean age $=19.4)$.

Stimuli and materials. The stimuli used in Experiment 3 are illustrated in Figure 4A. Like in Experiment 2, three fixation dots were to indicate where stimuli might appear. The three block types were Orientation-, Location-,and Both-blocks. In Orientation-blocks, we presented gabor stimuli with a spatial frequency of 1.8 cycles $/{ }^{\circ}$ and a two-dimensional standard deviation of $1.22^{\circ}$, rotated either $45^{\circ}$ clockwise or counterclockwise. In Location-blocks, the stimulus was an open white circle $6.11^{\circ}$ in 
diameter, which would appear on the left or on the right of the screen. The same circle was presented in the center of the screen for reference-trials and practice trials. In Both-blocks, a gabor similar to the one in the Orientation-blocks was presented, either on the left or on the right. The visible area of the gabor stimulus was similar in size to the circle used in Location-blocks.

Design and Procedure. The presentation sequence of a single trial was identical to Experiment 2. Blocks now had 48 trials which is somewhat more, in an attempt to maximize power while keeping the experiment under 1 hour. The entire experiment took approximately 55-60 minutes.

Statistical analyses. The statistical procedure was in every way identical to that of Experiments 1 and 2 .

\section{Results}

The results of Experiment 3 (Figure $4 \mathrm{~B} \& \mathrm{C}$ ) appear to be highly similar to those of Experiment 1. In blocks where the location of the markers changed, these changes have a pronounced effect on perceived duration. However, markers that changed in terms of their orientation seemed to have no such effects on time perception. Using the hypothesis-driven GLMM, we found that Tukey HSD tests on the change-same contrast across block types indicated that there was indeed a significant change bias in Location-blocks $(z=7.90, p<0.001)$ and Both-blocks $(z=9.03, p<0.001)$ but not in Orientation-blocks $(z=0.70, p=0.481)$. The same results were found with a GLMM where duration was coded by factorial bins (Orientation: $z=0.42, p<0.676$; Location: $z=7.62, p<0.001$; Both: $z=9.16 ; p<0.001)$. Model comparisons again supported the assumptions of the hypothesis driven model: we again found evidence that the change bias was constant across intervals $\left(\triangle B I C=-7.43 ; 1 / B F=41.06 ; \chi^{2}(1)=2.25 ; p=0.133\right)$, and that temporal sensitivity was not different across block types $\left(\triangle B I C=-13.84 ; 1 / B F>1000 ; \chi^{2}(2)=5.53 ; p=0.063\right)$. 


\section{Figure 4}

Stimuli and results in Experiment 3

\section{Experiment 3}
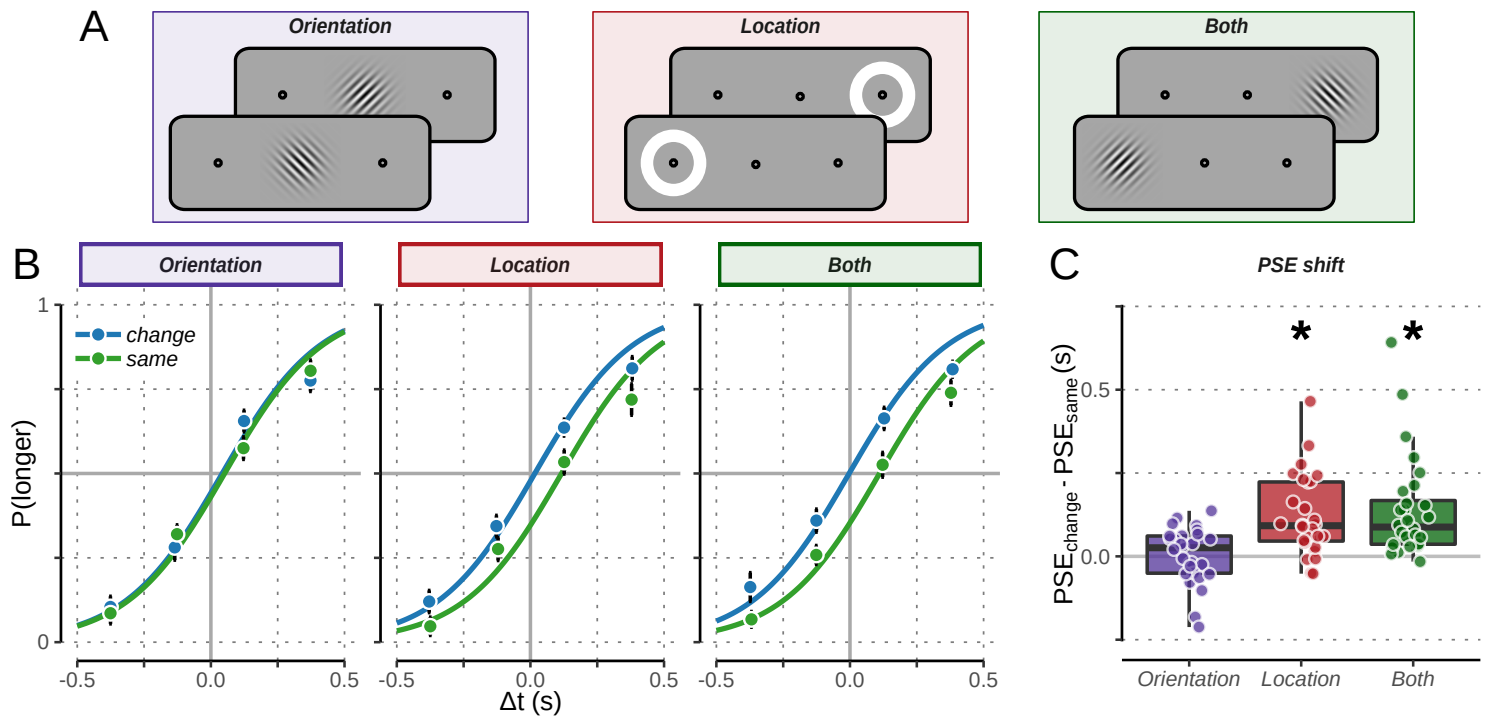

Note. cf. Figure 2, A Schematic illustration of the stimuli used as start- and end marker in different block types. B The GLMM fits again show a change bias in blocks where marker location changed, but not in Orientation-blocks. C PSE-differences computed from individual psychometric curves show the same result: no overall PSE-shifts were found in Orientation-blocks, while there was in the other two blocks.

Data-driven analyses of the PSE-shifts for each participant for each condition (Figure $4 \mathrm{C}$ ) corroborated these conclusions. We found strong evidence for a positive shift in PSE's in Location-blocks $(t(27)=-5.31 ; p<0.001 ; B F>1000)$ as well as in Both-blocks $(t(27)=-4.84, p<0.001, B F=524.75)$, but PSE's were found not differ in Orientation-blocks $(t(27)=-0.22 ; p=0.414 ; 1 / B F=4.88)$. Pairwise comparisons further highlighted how the change bias differed across block types: that is, the bias was higher in Location-blocks than Orientation-blocks $(t(27)=-3.94 ; p<0.001 ; B F=59.53)$, and higher in Both-Blocks than in Orientation-blocks $(t(27)=-4.21 ; p<0.001 ; B F=113.25)$, but did not seem to differ between Location- and Both-blocks 
$(t(27)=0.81 ; p=0.426 ; 1 / B F=3.70)$.

\section{Discussion}

Experiment 3 demonstrated that a change in the location of a gabor stimulus affected time perception, but that changes in the orientation of the gabor stimulus alone had no effect on perceived durations. The effect of orientation changes on neural responses in early visual areas like V1 is well established, and yet it seems that such effects did not modulate participants' perception of time. Note that this observation is in line with earlier

work with oddball stimuli (Schindel et al., 2011) where it was demonstrated that changing the eye of origin of the visual stimulus similarly did not affect perceived duration. This was taken as evidence against a strong reading of the magnitude coding hypothesis, which led to the conclusion that oddball effects are not the result of changes in sensory responses. The results of Experiments 1-3 argue for a more nuanced interpretation of those results. They support the idea of selectivity in magnitude coding effects as outlined in the introduction: repetition suppression in some feature dimensions has a more pronounced effect on the perception of time than in other feature dimensions.

Within the context of that hypothesis, the results so far are consistent with magnitude coding theories and thereby support the hypothesis that there are functional interactions between the visual representation of space, time, and stimulus size. Experiment 4 was designed to test this hypothesis further, and to investigate whether time perception is affected by changes in numerosity between stimulus markers.

\section{Experiment 4: Luminance and Numerosity}

In the introduction, the perception of time was characterized as a feat of generative perception, highlighting how vastly differing sensory input can give rise to the same sense of duration. To an extent, the same reasoning applies to the perception of numerosity. Yet, it seems that cortical areas, including parietal cortex, demonstrate selectivity to the number of stimuli that is perceived, largely independent of the visual appearance of these stimuli 
(Harvey et al., 2013; Walsh, 2003). In theories that postulate a shared representation among different quantities, this representation of numerosity is often included. Therefore, Experiment 4 explored whether changes in numerosity affect the perception of time.

One problem that 'quantity coding' theories face, is that the dimensions that are assumed to have a shared representation may be hard to manipulate independently in an orthogonal fashion. For example, in Experiment 2, we manipulated stimulus size, but in doing so this also affected the overall luminance of the presented stimuli. In manipulating numerosity, Harvey et al. (2013) used size-controlled stimuli, which equated the total surface area and thereby the luminance of the presented stimuli. Here, we will use the same approach, but also add an explicit control to test whether luminance changes affect perceived durations. Again, we used three block types: one with a single stimulus that changed its luminance (drastically) from white to black, one with numerosity changes from two to seven stimuli, controlled for luminance; and one where we manipulated the numerosity, with two or seven stimuli, without controlling for luminance (with stimuli of the same size in both conditions - which was in a sense comparable to the Both-blocks used in Experiments 1-3).

\section{Methods}

The design, procedure and analyses of Experiment 4 were almost identical to that of Experiment 3, differing only with regards to the stimuli that were used. Here, we primarily focus on aspects in which Experiment 4 differed from Experiment 3.

Participants. The protocol for Experiment 4 was approved by the EC BSS (internal review code: PSY-1920-S-0190). For Experiment 4, data were collected from 32 participants, of which 2 datasets were removed as one participant did not complete the experiment and another used over 20 minutes more than all other participants. None of the participants were excluded for bad performance. The remaining sample consisted of 30 participants (20 female, ages $18-29$, Mean age $=20.1$ ) 
Stimuli and materials. For consistency with Experiments 2 and 3, three fixation dots were presented throughout the trial, even though all marker stimuli in Experiment 4 were presented at the center of the screen. Participants were told they could ignore the lateral dots. For reference- and practice trials, onset- and offset markers were central, open white circles identical to reference trials in Experiment 3 (6.11 diameter). Figure $5 \mathrm{~A}$ illustrates the marker stimuli used in experimental trials. In Luminance-blocks, a filled circle of the same size was used, which could be either black or white. There were two types of Numerosity-blocks, and in both, we presented either two or seven white filled circles at fixed positions around fixation. In 'equiluminant' Numerosity-blocks, the size of these circles was adjusted such that the total surface area, and thereby the luminance, of all circles together was the same as the single circle in the Luminance-blocks:

$\left(4.32^{\circ}\right.$ diameter with two circles, $2.31^{\circ}$ diameter with seven circles). In 'normal' Numerosity blocks (hereafter named 'Numerosity-blocks'), circles had a fixed size, with an average total surface that was the same as the circles in Luminance-blocks (2.88 diameter).

Design and Procedure. Like Experiment 3, trials were distributed over twelve blocks of 48 trials each. The experiment took 55-60 minutes in total.

Statistical analyses. The statistical procedure was in every way identical to that of Experiments 1-3.

\section{Results}

Figure $5 \mathrm{~B}$ depicts the hypothesis-driven GLMM fits to the data of Experiment 4. The fits suggest no difference between same- and change-trials in Luminance-blocks, but a consistent, albeit small change bias effect for both Numerosity-block types. The pairwise contrasts per block type indeed show that there was a significant difference between sameand change-trials in equiluminant Numerosity-blocks $(z=2.08 ; p=0.037)$ as well as in Numerosity-blocks $(z=2.47 ; p=0.014)$, but not in Luminance-blocks $(z=0.967 ; p=0.334)$. Assessment of this contrast in a GLMM with duration coded as a 


\section{Figure 5}

Stimuli and results in Experiment 4

\section{Experiment 4}

A
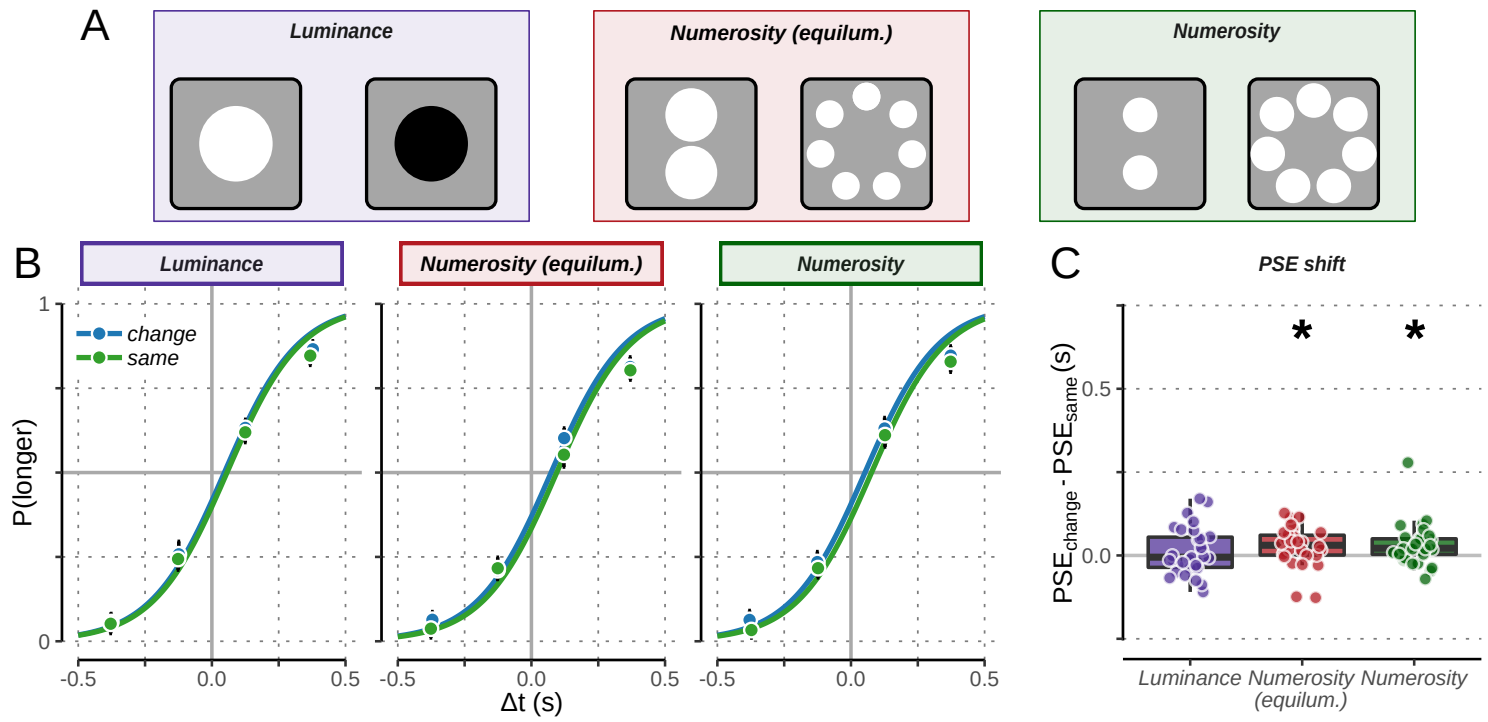

Note. cf. Figure2, A Schematic illustration of the stimuli used as start- and end marker in different block types. B The GLMM fits show relatively small PSE-shifts in both Numerosity-blocks, but no compelling shifts in the Luminance-blocks. C Individual curve fits showed small but consistent PSE-shifts in both Numerosity-blocks. In Luminance-blocks, evidence against such a positive shift was found.

factorial predictor yielded highly similar statistics (Luminance: $z=1.21, p=0.227$;

Numerosity (equiluminant): $z=1.97, p=0.049$; Numerosity: $z=2.23, p=0.026$ ). Model comparisons suggested that the assumptions of the hypothesis-driven model were justified. That is, there was strong evidence that the change bias did not differ across durations $\left(\triangle B I C=-9.68 ; 1 / B F=126.47 ; \chi^{2}(1)=0.073 ; p=0.78\right)$ and that duration sensitivity, indexed by the slope of the psychometric curve, was not different across blocks $\left(\triangle B I C=-15.75 ; 1 / B F>1000 ; \chi^{2}(2)=3.76 ; p=0.15\right)$.

Analyses based on the PSE shifts per block type (Figure $5 \mathrm{C}$ ) corroborated the findings of the GLMM analysis. We found evidence, albeit inconclusive, for a positive shift 
in PSE's in Equiluminant Numerosity blocks $(t(29)=-2.38 ; p=0.009 ; B F=2.63)$ and stronger evidence for such a shift in non-controlled Numerosity blocks $(t(29)=-2.71 ; p=0.006 ; B F=4.12)$. Conversely, for Luminance blocks, we found evidence against such a shift in PSE $(t(29)=-1.03 ; p=0.157 ; 1 / B F=3.19)$. These tests suggest that a change bias was present in blocks with numerosity changes, but not in blocks where only luminance was manipulated. We note, however, that pairwise comparisons of the change bias magnitude across blocks did not conclusively support this interpretation: all contrasts gave moderate evidence that there was no difference across blocks (all $-0.97<t(29)<0.31$;all $p>0.339$; all $3.34<1 / B F<4.92$ ).

Taken together, the results of Experiment 4 were less unequivocal than those of Experiments 1-3. Both the planned comparisons within the GLMM and the data-driven tests of PSE-shifts point to a change bias in both types of Numerosity-blocks, and no such bias in the Luminance-blocks. However, none of the statistical tests compellingly shows that change bias effects differed across block types. Furthermore, numerical trends indicated that the change biases in Experiment 4 might have been caused by an additive effect of changes in numerosity in concert with changes in luminance, and that both might have had an additive effect. We therefore chose to construct a more direct test to evaluate whether changes in numerosity, changes in luminance, or both affect time perception, similar to the follow-up analysis for Experiment 2. Once again, we split the variable 'trial type' (same/change) into two separate variables, one indicating 'numerosity changes' and one indicating 'luminance changes'. Subsequently, we tested whether a base model that included ' $\Delta t$ ' and 'block type' as main effects, would be improved by the addition of 'luminance change', 'numerosity change' or both. The results showed that the best model was one that only included an effect of 'numerosity change', which was strongly preferred over models that either only included an effect of 'luminance change' $(\triangle B I C=4.43 ; B F=9.27$; no likelihood ratio test possible $)$ or a predictor term for both $\left(\triangle B I C=-8.87 ; 1 / B F=84.71 ; \chi^{2}(1)=0.88 ; p=0.349\right)$, and was similarly preferred over 
the original, hypothesis-driven model

$\left(\triangle B I C=-18.52 ; 1 / B F>1000 ; \chi^{2}(2)=1.00 ; p=0.608\right)$. These results lend further support to the conclusion that changes in luminance did not affect time perception, whereas changes in numerosity do.

\section{Discussion}

The results of Experiment 4 lend further support to the hypothesis that magnitude coding effects show feature selectivity: change bias effects on time perception were found for changes in numerosity, but not for changes in luminance. The observation that a large change in luminance, from white to black stimuli and vice versa, did not have robust effects on time perception are in line with the findings of Experiment 3: They further corroborate the conclusion that changes affecting early stages of visual processing do not necessarily affect time perception (cf. Schindel et al., 2011). In similar vein, the change bias was found in response to changes in numerosity. This suggests that the change bias can be the result of changes in more abstract, high-level stimulus properties that are the result of generative perception. Moreover, it further points to a tight link between the change bias effects and theories that propose a shared neural representation for time, space, and quantities in different feature dimensions (Bueti et al., 2008, Dehaene \& Brannon, 2010; Harvey et al., 2015; Walsh, 2003).

\section{General Discussion}

In the present study, we investigated how the sensory experience of a range of stimulus features affects the perception of time. To this end, we measured participants' perception of the time between two briefly presented markers, which could either be the same or constitute a fully predictable change. Across four experiments, we tested the effects of changes in six dimensions: Location, Size, Numerosity, Face identity, Orientation and Luminance. The results indicate that for the first three of those dimensions, changes between the start- and end-marker of an interval resulted in a dilation of perceived 
duration compared to trials where the start- and end-marker were the same. These findings show that the 'change bias' that we found in earlier work (Kruijne et al., 2021) is a general finding that is not limited to changes in location. At the same time, the results suggest that the change bias shows selectivity, as changes in Faces, Orientation and Luminance did not affect time perception in the same way. These results may suggest that the sensory processing of these features does not relate to the computation of a temporal percept in the same way as Location, Size and Numerosity do.

What neural mechanisms underlie these change bias effects? In earlier work, we measured EEG-responses and pupil responses to stimuli where markers changed location (Kruijne et al., 2021). These physiological markers suggested that a stimulus at a repeated location resulted in an attenuation of its sensory response through repetition suppression, and that the magnitude of the sensory response to the offset of the interval played a causal role in the perception of its duration (cf. Mayo \& Sommer, 2013). The present study supports this interpretation: Repetitions and changes in other stimulus dimensions were expected to produce neural repetition suppression effects as well, and indeed, repetitions and switches in these dimensions evoked similar biases.

However, change biases were not found for all tested stimulus dimensions. The absence of change biases for several feature dimensions seems at odds with the strong reading of sensory magnitude coding hypothesis, which would predict that any sensory change could affect time perception. Based on this strong reading, one interpretation of our results is that the sensory effects of changes in location, size and numerosity are simply greater than for changes in faces, orientations and luminance. However, note that Bayesian evidence in these latter three dimensions generally supported the absence of any change bias. Moreover, in particular for changes in faces and orientation, their effects on both lateand early-stage sensory responses have been well-characterized and are known to be substantial (Boynton \& Finney, 2003 Larsson \& Smith, 2012; Summerfield et al., 2008; Summerfield et al., 2011; Tang et al., 2018). Rather, we believe that our results call for a 
more nuanced view of the magnitude coding hypothesis, and reveal that repetition suppression effects in some sensory pathways does not affect time perception. We interpret these results as indicating selectivity in the sensory neural pathways involved in the computation of a temporal percept, where only those neurons causally involved in computing temporal information will show temporal biases when affected by neural repetition suppression. As such, the change bias paradigm may offer a psychophysical tool to dissect the sensory pathways that are leading in time perception.

Of particular interest is the extent to which the present results are at odds with other findings that have been taken to reflect magnitude coding effects on time perception. A classical finding, for example, is that brighter- and louder stimuli are perceived as lasting longer than more subdued stimuli (Xuan et al., 2007). This observation is often interpreted as reflecting how the magnitude of the sensory response, in this case driven by luminance or loudness, causally modulates in time perception. In the present results, we found that changes in luminance did not affect time perception the way changes in other stimulus dimensions did, which may suggest that luminance may not play a causal role in the perception of time. Instead, it may be that the effects on time perception reported in earlier work may be the result of the indirect effects of heightened states of arousal or surprise 2 , Similar reasoning applies to a vast range of studies that have explored the effects of different kinds of oddball stimuli on time perception - for example how oddball stimuli that appear to be 'looming' have a more pronounced effect on time perception than stimuli that are 'receding' (Tse et al., 2004; Wassenhove et al., 2008). While these results may indeed reflect differential effects of different sensory properties on time perception, the

\footnotetext{
${ }^{2}$ In a separate analysis, we tested whether physical properties of the stimuli themselves might have affected the present results. That is, we tested whether the identity of marker stimuli on same-trials affected the rate of 'longer'-responses using a GLMM comparable to the hypothesis-driven change-bias analysis. We did so for Size-blocks in Experiment 2, and for Luminance- and Equiluminant Numerosity-blocks in Experiment 4. For all three block types, trends were in the expected direction, but no significant differences between different stimulus types were detected (all $z<0.73$; all $p>0.47$ )
} 
oddball design will generally have the inherent shortcoming that it is difficult to control for variability in surprise or arousal evoked by the statistical properties of the sequence (Pariyadath \& Eagleman, 2008, Sadeghi et al., 2011). To overcome this shortcoming, studies need to either design stimulus sequences that decouple contextual predictability from stimulus repetition (Cai et al., 2015; Wehrman et al., 2018), or investigate repetitions in a design that does not rely on stimulus sequences to build up contextual expectations.

The effects of stimulus repetition have only rarely been studied in isolation, outside of the context of stimulus sequences that also vary their degree of predictability: In one study (Matthews, 2011a), a temporal dilation effect was reported in response to changes in size, which is comparable to our findings in Experiment 2. Another study (Noguchi \& Kakigi, 2006) did not make use of start- and end-markers, but rather manipulated the relation between the standard- and comparison stimulus, both presented on every trial. The to-be-timed stimuli were arrowheads that pointed either to the left or to the right of fixation, which could be the same or opposite direction for the standard- or comparison interval. The results point to temporal dilation caused by changes, which is likely to have had very similar underpinnings to the change bias effects for location that we have reported in Experiments 1-3. Finally, another study by Matthews (2011b) similarly manipulated changes and repetitions between standard- and comparison stimuli presented on individual trials (similar to Noguchi \& Kakigi, 2006). In this study, stimuli were real-life color photographs which could change or repeat, and switches were found to dilate the perception of time. Consequentially, a range of stimulus features would have changed from one picture to the next, which may have included the size, location and numerosity of the objects in these photographs. Of note, in all three of these studies, changes and repetitions were not fully predictable, like they were in the present experiments. Therefore, the effects of changing stimuli might still have comprised both sensory effects as well as effects on the level of surprise they evoke. Another notable difference is that the ranges of durations tested in these studies were almost all shorter than 1 second. The findings of the present 
study illustrate that sensory repetitions and changes can affect the perception of duration well in the suprasecond range.

Regardless of how the present study compares to other studies on the effects of stimulus repetition, expectations, and sensory magnitude on the perception of time, our results reveal an interesting inherent dissociation of how temporal information is informed by different visual feature dimensions. That is, the repetitions and changes of Location, Size and Numerosity were found to have a pronounced effect on time perception whereas other visual features did not. This particular division among the six feature dimensions explored here aligns well with neuroimaging studies on the cortical representation of time, space, and number (Dehaene \& Brannon, 2010; Harvey et al., 2013, Harvey et al., 2020). In particular, these psychophysical results closely align with proposals that areas including parietal cortex serve as an integrative hub that is involved in representing quantities of different stimulus dimensions including size, duration, and number (Bueti et al., 2008; Bueti \& Walsh, 2009; Walsh, 2003). If neurons in parietal cortex indeed display multiplexed tuning for different quantities, it is feasible that when their response is affected by neural repetition suppression it will have a pronounced effect on the representation of other stimulus dimensions. This idea is additionally supported by findings regarding duration after-effects, the phenomenon that after repeated presentation of the same duration, subsequent temporal judgments are skewed away from the adapter duration, suggesting the existence of 'duration channels' (Becker \& Rasmussen, 2007; Heron et al., 2012; Maarseveen et al., 2017; Walker et al., 1981). Recent research on such duration adaptation related this phenomenon to changing neural responses in the parietal cortex, suggesting that this is a key site for representing durations in a manner that is sensitive to the magnitude of neural responses (Hayashi et al., 2015, Hayashi \& Ivry, 2020). Future neuroimaging studies might therefore similarly be able to relate the change biases of the present study to response attenuation in the parietal cortex. However, we stress that these effects may not be limited to one locus: Other studies have suggested that duration 
channels may form a hierarchical, vastly distributed network with various potential sites of interaction between the representation of size, location, numerosity and duration (Harvey et al., 2020; Harvey et al., 2015).

Taken together, the present results illustrate that change biases on time perception can be found for several, though not all feature dimensions. The characteristics of these bias effects, together with earlier work demonstrating the electrophysiological characteristics of this bias strongly point to the involvement of neural repetition suppression and thereby support the magnitude coding hypothesis. At the same time, the present results offer important constraints for this hypothesis, as they indicate that the effects of sensory responses on time perception are selective, with certain features contributing more strongly to the temporal percept than others. Importantly, these results also illustrate that the classical oddball paradigm may not be an appropriate paradigm to measure this selectivity, as in most of these studies repetition suppression is correlated with expectation suppression and arousal effects. As these psychophysical findings converge with recent neuroimaging results, they together begin to unveil how a malleable sense of subjective time is formed by our sensory apparatus, shaped by the stimuli used to present it. 


\section{References}

Acerbi, L., Wolpert, D. M., \& Vijayakumar, S. (2012). Internal representations of temporal statistics and feedback calibrate motor-sensory interval timing. PLOS Computational Biology, 8(11), e1002771. https://doi.org/10.1371/journal.pcbi.1002771

Baayen, R. H., Davidson, D. J., \& Bates, D. M. (2008). Mixed-effects modeling with crossed random effects for subjects and items. Journal of Memory and Language, 59(4), 390-412. https://doi.org/10.1016/j.jml.2007.12.005

Bausenhart, K. M., Dyjas, O., \& Ulrich, R. (2014). Temporal reproductions are influenced by an internal reference: Explaining the vierordt effect. Acta Psychologica, 147, 60-67. https://doi.org/10.1016/j.actpsy.2013.06.011

Beck, D. M., \& Kastner, S. (2007). Stimulus similarity modulates competitive interactions in human visual cortex. Journal of Vision, 7(2), 19-19. https://doi.org/10.1167/7.2.19

Becker, M. W., \& Rasmussen, I. P. (2007). The rhythm aftereffect: Support for time sensitive neurons with broad overlapping tuning curves. Brain and cognition, 64(3), $274-281$.

Boynton, G. M., \& Finney, E. M. (2003). Orientation-specific adaptation in human visual cortex. Journal of Neuroscience, 23(25), 8781-8787. https://doi.org/10.1523/JNEUROSCI.23-25-08781.2003

Brysbaert, M., \& Stevens, M. (2018). Power analysis and effect size in mixed effects models: A tutorial. Journal of Cognition, 1(1), 9. https://doi.org/10.5334/joc.10

Bueti, D., Bahrami, B., \& Walsh, V. (2008). Sensory and association cortex in time perception. Journal of Cognitive Neuroscience, 20(6), 1054-1062.

https://doi.org/10.1162/jocn.2008.20060 
Bueti, D., \& Walsh, V. (2009). The parietal cortex and the representation of time, space, number and other magnitudes. Philosophical Transactions of the Royal Society B: Biological Sciences, 364(1525), 1831-1840. https://doi.org/10.1098/rstb.2009.0028

Bueti, D., Walsh, V., Frith, C., \& Rees, G. (2007). Different brain circuits underlie motor and perceptual representations of temporal intervals. Journal of Cognitive Neuroscience, 20(2), 204-214. https://doi.org/10.1162/jocn.2008.20017

Buhusi, C. V., \& Meck, W. H. (2005). What makes us tick? functional and neural mechanisms of interval timing. Nature Reviews Neuroscience, 6(10), 755-765. https://doi.org/10.1038/nrn1764

Buia, C., \& Tiesinga, P. (2006). Attentional modulation of firing rate and synchrony in a model cortical network. Journal of Computational Neuroscience, 20(3), 247-264. https://doi.org/10.1007/s10827-006-6358-0

Cai, M. B., Eagleman, D. M., \& Ma, W. J. (2015). Perceived duration is reduced by repetition but not by high-level expectation. Journal of Vision, 15(13), 19-19. https://doi.org/10.1167/15.13.19

Cho, J. R., Treweek, J. B., Robinson, J. E., Xiao, C., Bremner, L. R., Greenbaum, A., \& Gradinaru, V. (2017). Dorsal raphe dopamine neurons modulate arousal and promote wakefulness by salient stimuli. Neuron, 94(6), 1205-1219.e8. https://doi.org/10.1016/j.neuron.2017.05.020

Chun, M. M., Golomb, J. D., \& Turk-Browne, N. B. (2011). A taxonomy of external and internal attention. Annual Review of Psychology, 62(1), 73-101. https://doi.org/10.1146/annurev.psych.093008.100427

Clark, A. (2013). Whatever next? predictive brains, situated agents, and the future of cognitive science. Behavioral and Brain Sciences, 36(03), 181-204. https://doi.org/10.1017/S0140525X12000477 
Coull, J. T., Cheng, R.-K., \& Meck, W. H. (2011). Neuroanatomical and neurochemical substrates of timing. Neuropsychopharmacology, 36(1), 3-25. https://doi.org/10.1038/npp.2010.113

Cousineau, D. (2005). Confidence intervals in within-subject designs: A simpler solution to loftus and masson's method. Tutorials in Quantitative Methods for Psychology, 1(1), 42-45. https://doi.org/10.20982/tqmp.01.1.p042

Dehaene, S., \& Brannon, E. M. (2010). Space, time, and number: A kantian research program. Trends in Cognitive Sciences, 14(12), 517-519. https://doi.org/10.1016/j.tics.2010.09.009

Droit-Volet, S., \& Meck, W. H. (2007). How emotions colour our perception of time. Trends in Cognitive Sciences, 11(12), 504-513. https://doi.org/10.1016/j.tics.2007.09.008

Eagleman, D. M. (2008). Human time perception and its illusions. Current Opinion in Neurobiology, 18(2), 131-136. https://doi.org/10.1016/j.conb.2008.06.002

Eagleman, D. M., \& Pariyadath, V. (2009). Is subjective duration a signature of coding efficiency? Philosophical Transactions of the Royal Society B: Biological Sciences, 364 (1525), 1841-1851. https://doi.org/10.1098/rstb.2009.0026

Ekman, G., Frankenhaeuser, M., Berglund, B., \& Waszak, M. (1969). Apparent duration as a function of intensity of vibrotactile stimulation. Perceptual and Motor Skills, $28(1), 151-156$.

Ernst, B., Reichard, S. M., Riepl, R. F., Steinhauser, R., Zimmermann, S. F., \& Steinhauser, M. (2017). The p3 and the subjective experience of time. Neuropsychologia, 103, 12-19. https://doi.org/10.1016/j.neuropsychologia.2017.06.033

Fang, F., Murray, S. O., Kersten, D., \& He, S. (2005). Orientation-tuned fmri adaptation in human visual cortex. Journal of Neurophysiology, 94 (6), 4188-4195. https://doi.org/10.1152/jn.00378.2005 
Fiorillo, C. D., Song, M. R., \& Yun, S. R. (2013). Multiphasic temporal dynamics in responses of midbrain dopamine neurons to appetitive and aversive stimuli. Journal of Neuroscience, 33(11), 4710-4725. https://doi.org/10.1523/JNEUROSCI.3883-12.2013

Friston, K. (2005). A theory of cortical responses. Philosophical Transactions of the Royal Society B: Biological Sciences, 360(1456), 815-836. https://doi.org/10.1098/rstb.2005.1622

Fung, B. J., Sutlief, E., \& Hussain Shuler, M. G. (2021). Dopamine and the interdependency of time perception and reward. Neuroscience $\&$ Biobehavioral Reviews, 125, 380-391. https://doi.org/10.1016/j.neubiorev.2021.02.030

Georghiades, A., Belhumeur, P., \& Kriegman, D. (2001). From few to many: Illumination cone models for face recognition under variable lighting and pose. IEEE Transactions on Pattern Analysis and Machine Intelligence, 23(6), 643-660. https://doi.org/10.1109/34.927464

Gibbon, J. (1977). Scalar expectancy theory and weber's law in animal timing. Psychological review, 84(3), 279.

Goldstone, S., Lhamon, W. T., \& Sechzer, J. (1978). Light intensity and judged duration. Bulletin of the Psychonomic Society, 12(1), 83-84. https://doi.org/10.3758/BF03329633

Green, P., \& MacLeod, C. J. (2016). Simr: An r package for power analysis of generalized linear mixed models by simulation. Methods in Ecology and Evolution, 7(4), 493-498. https://doi.org/https://doi.org/10.1111/2041-210X.12504

Grill-Spector, K., Henson, R., \& Martin, A. (2006). Repetition and the brain: Neural models of stimulus-specific effects. Trends in Cognitive Sciences, 10(1), 14-23. https://doi.org/10.1016/j.tics.2005.11.006 
Hansen, B. J., \& Dragoi, V. (2011). Adaptation-induced synchronization in laminar cortical circuits. Proceedings of the National Academy of Sciences, 108(26), 10720-10725. https://doi.org/10.1073/pnas.1102017108

Harvey, B. M., Klein, B. P., Petridou, N., \& Dumoulin, S. O. (2013). Topographic representation of numerosity in the human parietal cortex. Science, 341(6150), 1123-1126. https://doi.org/10.1126/science.1239052

Harvey, B. M., Dumoulin, S. O., Fracasso, A., \& Paul, J. M. (2020). A network of topographic maps in human association cortex hierarchically transforms visual timing-selective responses. Current Biology, 30(8), 1424-1434.e6. https://doi.org/10.1016/j.cub.2020.01.090

Harvey, B. M., Fracasso, A., Petridou, N., \& Dumoulin, S. O. (2015). Topographic representations of object size and relationships with numerosity reveal generalized quantity processing in human parietal cortex. Proceedings of the National Academy of Sciences, 112(44), 13525-13530. https://doi.org/10.1073/pnas.1515414112

Hayashi, M. J., Ditye, T., Harada, T., Hashiguchi, M., Sadato, N., Carlson, S., Walsh, V., \& Kanai, R. (2015). Time adaptation shows duration selectivity in the human parietal cortex. PLOS Biology, $13(9)$, e1002262. https://doi.org/10.1371/journal.pbio.1002262

Hayashi, M. J., \& Ivry, R. B. (2020). Duration selectivity in right parietal cortex reflects the subjective experience of time. Journal of Neuroscience, 40(40), 7749-7758. https://doi.org/10.1523/JNEUROSCI.0078-20.2020

Henson, R. N. (2016). Repetition suppression to faces in the fusiform face area: A personal and dynamic journey. Cortex, 80, 174-184.

Heron, J., Aaen-Stockdale, C., Hotchkiss, J., Roach, N. W., McGraw, P. V., \& Whitaker, D. (2012). Duration channels mediate human time perception. Proceedings of the Royal Society B: Biological Sciences, 279(1729), 690-698. https://doi.org/10.1098/rspb.2011.1131 
Ishai, A., Bikle, P. C., \& Ungerleider, L. G. (2006). Temporal dynamics of face repetition suppression. Brain Research Bulletin, 70(4-6), 289-295.

Jazayeri, M., \& Shadlen, M. N. (2010). Temporal context calibrates interval timing. Nature Neuroscience, 13(8), 1020-1026. https://doi.org/10.1038/nn.2590

Kanayet, F. J., Mattarella-Micke, A., Kohler, P. J., Norcia, A. M., McCandliss, B. D., \& McClelland, J. L. (2017). Distinct representations of magnitude and spatial position within parietal cortex during number-space mapping. Journal of Cognitive Neuroscience, 30(2), 200-218. https://doi.org/10.1162/jocn\_a \_01199

Kanizsa, G. (1955). 1987"quasi-perceptual margins in homogeneously stimulated fields", translation into english in the perception of illusory contours eds s petry. GE Meyer (1987, New York: Springer) pp, 40-49.

Kok, P., Jehee, J. F. M., \& de Lange, F. P. (2012). Less is more: Expectation sharpens representations in the primary visual cortex. Neuron, 75(2), 265-270. https://doi.org/10.1016/j.neuron.2012.04.034

Kruijne, W., Olivers, C. N. L., \& van Rijn, H. (2021). Neural repetition suppression modulates time perception: Evidence from electrophysiology and pupillometry. Journal of Cognitive Neuroscience, 1-20. https://doi.org/10.1162/jocn\_a \_01705

Lake, J. I., \& Meck, W. H. (2013). Differential effects of amphetamine and haloperidol on temporal reproduction: Dopaminergic regulation of attention and clock speed. Neuropsychologia, 51 (2), 284-292. https://doi.org/10.1016/j.neuropsychologia.2012.09.014

Lambrechts, A., Walsh, V., \& Wassenhove, V. v. (2013). Evidence accumulation in the magnitude system. PLOS ONE, 8(12), e82122. https://doi.org/10.1371/journal.pone.0082122

Larsson, J., \& Smith, A. T. (2012). Fmri repetition suppression: Neuronal adaptation or stimulus expectation? Cerebral Cortex, 22(3), 567-576. https://doi.org/10.1093/cercor/bhr119 
Learned-Miller, E., Huang, G. B., RoyChowdhury, A., Li, H., \& Hua, G. (2016). Labeled faces in the wild: A survey (M. Kawulok, M. E. Celebi, \& B. Smolka, Eds.). In M. Kawulok, M. E. Celebi, \& B. Smolka (Eds.), Advances in face detection and facial image analysis, Cham, Springer International Publishing.

Lejeune, H., \& Wearden, J. H. (2009). Vierordt's the experimental study of the time sense (1868) and its legacy. European Journal of Cognitive Psychology, 21 (6), 941-960. https://doi.org/10.1080/09541440802453006

Lenth, R. (2020). Emmeans: Estimated marginal means, aka least-squares means [R package version 1.4.8]. $\mathrm{R}$ package version 1.4.8.

Maarseveen, J., Hogendoorn, H., Verstraten, F. A. J., \& Paffen, C. L. E. (2017). An investigation of the spatial selectivity of the duration after-effect. Vision Research, 130, 67-75. https://doi.org/10.1016/j.visres.2016.11.003

Maaß, S. C., Schlichting, N., \& van Rijn, H. (2019). Eliciting contextual temporal calibration: The effect of bottom-up and top-down information in reproduction tasks. Acta Psychologica, 199, 102898. https://doi.org/10.1016/j.actpsy.2019.102898

Martin, B., Wiener, M., \& Wassenhove, V. v. (2017). A bayesian perspective on accumulation in the magnitude system. Scientific Reports, 7(1), 1-14. https://doi.org/10.1038/s41598-017-00680-0

Matell, M. S., Kim, J. S., \& Hartshorne, L. (2014). Timing in a variable interval procedure: Evidence for a memory singularity. Behavioural Processes, 101, 49-57. https://doi.org/10.1016/j.beproc.2013.08.010

Mather, M., Clewett, D., Sakaki, M., \& Harley, C. W. (2016). Norepinephrine ignites local hotspots of neuronal excitation: How arousal amplifies selectivity in perception and memory. Behavioral and Brain Sciences, 39. https://doi.org/10.1017/S0140525X15000667 
Mathôt, S., Schreij, D., \& Theeuwes, J. (2012). Opensesame: An open-source, graphical experiment builder for the social sciences. Behavior Research Methods, 44(2), 314-324. https://doi.org/10.3758/s13428-011-0168-7

Matthews, W. J. (2011a). Can we use verbal estimation to dissect the internal clock? differentiating the effects of pacemaker rate, switch latencies, and judgment processes. Behavioural Processes, 86(1), 68-74. https://doi.org/10.1016/j.beproc.2010.09.006

Matthews, W. J. (2011b). Stimulus repetition and the perception of time: The effects of prior exposure on temporal discrimination, judgment, and production. PLOS ONE, 6(5), e19815. https://doi.org/10.1371/journal.pone.0019815

Matthews, W. J. (2015). Time perception: The surprising effects of surprising stimuli. Journal of Experimental Psychology: General, 144 (1), 172-197. https://doi.org/10.1037/xge0000041

Matthews, W. J., Terhune, D. B., van Rijn, H., Eagleman, D. M., Sommer, M. A., \& Meck, W. H. (2014). Subjective duration as a signature of coding efficiency: Emerging links among stimulus repetition, predictive coding, and cortical gaba levels. Timing $\&$ Time Perception Reviews, 1.

Mayo, J. P., \& Sommer, M. A. (2013). Neuronal correlates of visual time perception at brief timescales. Proceedings of the National Academy of Sciences, 110(4), 1506-1511. https://doi.org/10.1073/pnas.1217177110

McGurk, H., \& MacDonald, J. (1976). Hearing lips and seeing voices. Nature, 264(5588), $746-748$.

Meck, W. H. (1986). Affinity for the dopamine d2 receptor predicts neuroleptic potency in decreasing the speed of an internal clock. Pharmacology Biochemistry and Behavior, 25 (6), 1185-1189. https://doi.org/10.1016/0091-3057(86)90109-7 
Meck, W. H. (2006). Neuroanatomical localization of an internal clock: A functional link between mesolimbic, nigrostriatal, and mesocortical dopaminergic systems. Brain Research, 1109 (1), 93-107. https://doi.org/10.1016/j.brainres.2006.06.031

Mikhael, J. G., \& Gershman, S. J. (2019). Adapting the flow of time with dopamine. APSselect, 6(5), 1748-1760. https://doi.org/10.1152/jn.00817.2018@apsselect.2019.6.issue-5

Morey, R. D. (2008). Confidence intervals from normalized data: A correction to cousineau (2005). Tutorials in Quantitative Methods for Psychology, 4(2), 61-64. https://doi.org/10.20982/tqmp.04.2.p061

Movshon, J. A., \& Van Sluyters, R. C. (1981). Visual neural development. Annual Review of Psychology, 32(1), 477-522. https://doi.org/10.1146/annurev.ps.32.020181.002401

Noguchi, Y., \& Kakigi, R. (2006). Time representations can be made from nontemporal information in the brain: An meg study. Cerebral Cortex, 16(12), 1797-1808. https://doi.org/10.1093/cercor/bhj117

Okamoto, H., \& Fukai, T. (2001). Neural mechanism for a cognitive timer. Physical Review Letters, 86(17), 3919-3922. https://doi.org/10.1103/PhysRevLett.86.3919

Oprisan, S. A., \& Buhusi, C. V. (2014). What is all the noise about in interval timing? Philosophical Transactions of the Royal Society B: Biological Sciences, 369(1637), 20120459. https://doi.org/10.1098/rstb.2012.0459

Pariyadath, V., \& Eagleman, D. M. (2008). Brief subjective durations contract with repetition. Journal of Vision, 8(16), 11-11. https://doi.org/10.1167/8.16.11

Paton, J. J., \& Buonomano, D. V. (2018). The neural basis of timing: Distributed mechanisms for diverse functions. Neuron, 98(4), 687-705. https://doi.org/10.1016/j.neuron.2018.03.045

Peirce, J. W. (2007). Psychopy-psychophysics software in python. Journal of Neuroscience Methods, 162(1-2), 8-13. https://doi.org/10.1016/j.jneumeth.2006.11.017 
Penton-Voak, I. S., Edwards, H., Percival, A., \& Wearden, J. H. (1996). Speeding up an internal clock in humans? effects of click trains on subjective duration. Journal of Experimental Psychology: Animal Behavior Processes, 22(3), 307.

Pérez, O., \& Merchant, H. (2018). The synaptic properties of cells define the hallmarks of interval timing in a recurrent neural network. Journal of Neuroscience, 38(17), 4186-4199. https://doi.org/10.1523/JNEUROSCI.2651-17.2018

Petzschner, F. H., \& Glasauer, S. (2011). Iterative bayesian estimation as an explanation for range and regression effects: A study on human path integration. Journal of Neuroscience, 31(47), 17220-17229. https://doi.org/10.1523/JNEUROSCI.2028-11.2011

Protopapa, F., Hayashi, M. J., Kulashekhar, S., Zwaag, W. v. d., Battistella, G., Murray, M. M., Kanai, R., \& Bueti, D. (2019). Chronotopic maps in human supplementary motor area. PLOS Biology, 17(3), e3000026. https://doi.org/10.1371/journal.pbio.3000026

R Core Team. (2020). R: A language and environment for statistical computing. $\mathrm{R}$ Foundation for Statistical Computing. Vienna, Austria.

Rao, R. P. N., \& Ballard, D. H. (1999). Predictive coding in the visual cortex: A functional interpretation of some extra-classical receptive-field effects. Nature Neuroscience, 2(1), 79-87. https://doi.org/10.1038/4580

Rouder, J. N., Speckman, P. L., Sun, D., Morey, R. D., \& Iverson, G. (2009). Bayesian t tests for accepting and rejecting the null hypothesis. Psychonomic Bulletin 8 Review, 16(2), 225-237. https://doi.org/10.3758/PBR.16.2.225

Sadeghi, N. G., Pariyadath, V., Apte, S., Eagleman, D. M., \& Cook, E. P. (2011). Neural correlates of subsecond time distortion in the middle temporal area of visual cortex. Journal of Cognitive Neuroscience, 23(12), 3829-3840.

https://doi.org/10.1162/jocn \_a \_00071 
Samaria, F. S., \& Harter, A. C. (1994). Parameterisation of a stochastic model for human face identification, In Proceedings of 1994 ieee workshop on applications of computer vision, IEEE.

Schindel, R., Rowlands, J., \& Arnold, D. H. (2011). The oddball effect: Perceived duration and predictive coding. Journal of Vision, 11(2), 17-17. https://doi.org/10.1167/11.2.17

Schultz, W. (2016). Dopamine reward prediction error coding. Dialogues in Clinical Neuroscience, 18(1), 23-32.

Schultz, W., Dayan, P., \& Montague, P. R. (1997). A neural substrate of prediction and reward. Science, $275(5306), 1593-1599$. https://doi.org/10.1126/science.275.5306.1593

Shankar, K. H., \& Howard, M. W. (2011). A scale-invariant internal representation of time. Neural Computation, 24(1), 134-193. https://doi.org/10.1162/NECO \_a\_00212

Simen, P., Balci, F., deSouza, L., Cohen, J. D., \& Holmes, P. (2011). A model of interval timing by neural integration. Journal of Neuroscience, 31(25), 9238-9253. https://doi.org/10.1523/JNEUROSCI.3121-10.2011

Soares, S., Atallah, B. V., \& Paton, J. J. (2016). Midbrain dopamine neurons control judgment of time. Science, 354(6317), 1273-1277. https://doi.org/10.1126/science.aah5234

Sohn, H., Narain, D., Meirhaeghe, N., \& Jazayeri, M. (2018). Bayesian computation through cortical latent dynamics. bioRxiv, 465419.

Stefanics, G., Kremláček, J., \& Czigler, I. (2014). Visual mismatch negativity: A predictive coding view. Frontiers in Human Neuroscience, 8. https://doi.org/10.3389/fnhum.2014.00666

Summerfield, C., Trittschuh, E. H., Monti, J. M., Mesulam, M.-M., \& Egner, T. (2008). Neural repetition suppression reflects fulfilled perceptual expectations. Nature Neuroscience, 11(9), 1004-1006. https://doi.org/10.1038/nn.2163 
Summerfield, C., Wyart, V., Mareike Johnen, V., \& de Gardelle, V. (2011). Human scalp electroencephalography reveals that repetition suppression varies with expectation. Frontiers in Human Neuroscience, 5. https://doi.org/10.3389/fnhum.2011.00067

Taatgen, N., \& van Rijn, H. (2011). Traces of times past: Representations of temporal intervals in memory. Memory $\&$ Cognition, 39(8), 1546-1560. https://doi.org/10.3758/s13421-011-0113-0

Tang, M. F., Smout, C. A., Arabzadeh, E., \& Mattingley, J. B. (2018). Prediction error and repetition suppression have distinct effects on neural representations of visual information (C. Summerfield, T. E. Behrens, P. Kok, \& H. Op de Beeck, Eds.). eLife, 7, e33123. https://doi.org/10.7554/eLife.33123

Thönes, S., von Castell, C., Iflinger, J., \& Oberfeld, D. (2018). Color and time perception: Evidence for temporal overestimation of blue stimuli. Scientific Reports, 8(1), 1688. https://doi.org/10.1038/s41598-018-19892-z

Todorovic, A., \& Lange, F. P. d. (2012). Repetition suppression and expectation suppression are dissociable in time in early auditory evoked fields. The Journal of Neuroscience, 32(39), 13389-13395. https://doi.org/10.1523/JNEUROSCI.2227-12.2012

Tootell, R. B., Silverman, M. S., Hamilton, S. L., Switkes, E., \& Valois, R. D. (1988). Functional anatomy of macaque striate cortex. v. spatial frequency. Journal of Neuroscience, 8(5), 1610-1624. https://doi.org/10.1523/JNEUROSCI.08-05-01610.1988

Tse, P. U., Intriligator, J., Rivest, J., \& Cavanagh, P. (2004). Attention and the subjective expansion of time. Perception \& Psychophysics, 66(7), 1171-1189. https://doi.org/10.3758/BF03196844

Ulrich, R., Nitschke, J., \& Rammsayer, T. (2006). Perceived duration of expected and unexpected stimuli. Psychological Research, 70(2), 77-87. https://doi.org/10.1007/s00426-004-0195-4 
van Rijn, H., Gu, B.-M., \& Meck, W. H. (2014). Dedicated clock/timing-circuit theories of time perception and timed performance (H. Merchant \& V. de Lafuente, Eds.). In H. Merchant \& V. de Lafuente (Eds.), Neurobiology of interval timing, New York, NY, Springer New York.

Vierordt, K. (1868). Der Zeitsinn nach Versuchen. H. Laupp.

Wagenmakers, E.-J. (2007). A practical solution to the pervasive problems ofp values. Psychonomic Bulletin \& Review, 14(5), 779-804.

https://doi.org/10.3758/BF03194105

Walker, J. T., Irion, A. L., \& Gordon, D. G. (1981). Simple and contingent aftereffects of perceived duration in vision and audition. Perception \& Psychophysics, 29(5), $475-486$.

Walsh, V. (2003). A theory of magnitude: Common cortical metrics of time, space and quantity. Trends in Cognitive Sciences, 7(11), 483-488. https://doi.org/10.1016/j.tics.2003.09.002

Wassenhove, V. v., Buonomano, D. V., Shimojo, S., \& Shams, L. (2008). Distortions of subjective time perception within and across senses. PLOS ONE, 3(1), e1437. https://doi.org/10.1371/journal.pone.0001437

Wearden, J. H., Williams, E. A., \& Jones, L. A. (2017). What speeds up the internal clock? effects of clicks and flicker on duration judgements and reaction time. Quarterly Journal of Experimental Psychology, 70(3), 488-503. https://doi.org/10.1080/17470218.2015.1135971

Wehrman, J. J., Wearden, J., \& Sowman, P. (2018). The expected oddball: Effects of implicit and explicit positional expectation on duration perception. Psychological Research. https://doi.org/10.1007/s00426-018-1093-5

Wiener, M., Turkeltaub, P., \& Coslett, H. B. (2010). The image of time: A voxel-wise meta-analysis. NeuroImage, $49(2), 1728-1740$. https://doi.org/10.1016/j.neuroimage.2009.09.064 
Xuan, B., Zhang, D., He, S., \& Chen, X. (2007). Larger stimuli are judged to last longer. Journal of Vision, 7(10), 2-2. https://doi.org/10.1167/7.10.2

Zelkind, I. (1973). Factors in time estimation and a case for the internal clock. The Journal of General Psychology, 88(2), 295-301. https://doi.org/10.1080/00221309.1973.9920735 